\title{
Thin Shell Implies Spectral Gap up to Polylog via a Stochastic Localization Scheme
}

\author{
Ronen Eldan*
}

August 4, 2018

\begin{abstract}
We consider the isoperimetric inequality on the class of high-dimensional isotropic convex bodies. We establish quantitative connections between two well-known open problems related to this inequality, namely, the thin shell conjecture, and the conjecture by Kannan, Lovász, and Simonovits, showing that the corresponding optimal bounds are equivalent up to logarithmic factors. In particular we prove that, up to logarithmic factors, the minimal possible ratio between surface area and volume is attained on ellipsoids. We also show that a positive answer to the thin shell conjecture would imply an optimal dependence on the dimension in a certain formulation of the Brunn-Minkowski inequality. Our results rely on the construction of a stochastic localization scheme for log-concave measures.
\end{abstract}

\section{Introduction}

The starting point of this paper is a conjecture by Kannan, Lovász, and Simonovits (in short, the KLS conjecture) about the isoperimetric inequality for convex bodies in $\mathbb{R}^{n}$. Roughly speaking, The KLS conjecture asserts that, up to a universal constant, the most efficient way to cut a convex body into two parts is with a hyperplane. To be more precise, given convex body $K \subset \mathbb{R}^{n}$ whose barycenter is at the origin, and a subset $T \subset K$ with $\operatorname{Vol}_{n}(T)=R \operatorname{Vol}_{n}(K)$, the KLS conjecture suggests that

$$
\operatorname{Vol}_{n-1}(\partial T \cap \operatorname{Int}(K)) \geq R C \inf _{\theta \in S^{n-1}} \operatorname{Vol}_{n-1}\left(K \cap \theta^{\perp}\right)
$$

for some universal constant $C>0$, whenever $R \leq \frac{1}{2}$. Here, $\operatorname{Vol}_{n-1}$ stands for the $(n-1)$-dimensional volume, $S^{n-1}$ is the unit sphere, $\theta^{\perp}$ is the hyperplane passing through the origin whose normal direction is $\theta$ and $\operatorname{Int}(K)$ is the interior of $K$.

The point of this paper is to reduce this conjecture to the case where $T$ is an ellipsoid, up to a logarithmic correction.

* Supported in part by the Israel Science Foundation and by a Marie Curie Grant from the Commission of the European Communities. 
In order to give a precise formulation of the KLS conjecture, we begin with some notation. A probability density $\rho: \mathbb{R}^{n} \rightarrow[0, \infty)$ is called log-concave if it takes the form $\rho=\exp (-H)$ for a convex function $H: \mathbb{R}^{n} \rightarrow \mathbb{R} \cup\{\infty\}$. A probability measure is log-concave if it has a log-concave density. The uniform probability measure on a convex body is an example for a logconcave probability measure, as well as, say, the gaussian measure in $\mathbb{R}^{n}$. A log-concave probability density decays exponentially at infinity, and thus has moments of all orders. For a probability measure $\mu$ on $\mathbb{R}^{n}$ with finite second moments, we consider its barycenter $b(\mu) \in \mathbb{R}^{n}$ and covariance matrix $\operatorname{Cov}(\mu)$ defined by

$$
b(\mu)=\int_{\mathbb{R}^{n}} x d \mu(x), \quad \operatorname{Cov}(\mu)=\int_{\mathbb{R}^{n}}(x-b(\mu)) \otimes(x-b(\mu)) d \mu(x)
$$

where for $x \in \mathbb{R}^{n}$ we write $x \otimes x$ for the $n \times n$ matrix $\left(x_{i} x_{j}\right)_{i, j=1, \ldots, n}$. A log-concave probability measure $\mu$ on $\mathbb{R}^{n}$ is isotropic if its barycenter lies at the origin and its covariance matrix is the identity matrix.

Given a measure $\mu$, Minkowski's boundary measure of a Borel set $A \subset \mathbb{R}^{n}$, is defined by,

$$
\mu^{+}(A)=\liminf _{\varepsilon \rightarrow 0^{+}} \frac{\mu\left(A_{\varepsilon}\right)-\mu(A)}{\varepsilon}
$$

where

$$
A_{\varepsilon}:=\left\{x \in \mathbb{R}^{n} ; \quad \exists y,|x-y| \leq \varepsilon\right\}
$$

is the $\varepsilon$-extension of $A$.

The main point of this paper is to find an upper bound for the constant,

$$
G_{n}^{-1}:=\inf _{\mu} \inf _{A \subset \mathbb{R}^{n}} \frac{\mu^{+}(A)}{\mu(A)}
$$

where $\mu$ runs over all isotropic log-concave measures in $\mathbb{R}^{n}$ and $A \subset \mathbb{R}^{n}$ runs over all Borel sets with $\mu(A) \leq \frac{1}{2}$.

The constant $G_{n}$ is known as the optimal inverse Cheeger constant. According to a result of Ledoux, $\left[\mathrm{L}\right.$, the quantity $G_{n}^{-2}$ is also equivalent, up to a universal constant, to the optimal spectral gap constant of isotropic log-concave measures in $\mathbb{R}^{n}$ (see (3) below). For an extensive review of this constant and equivalent formulations, see [Mil1]. One property of $G_{n}$ of particular importance in this note is,

$$
\frac{1}{C} G_{n}^{2} \leq \sup _{\mu} \sup _{\varphi} \frac{\int \varphi^{2} d \mu}{\int|\nabla \varphi|^{2} d \mu} \leq C G_{n}^{2}
$$

Where $\mu$ runs over all isotropic log-concave measures and $\varphi$ runs over all smooth enough functions with $\int \varphi d \mu=0$ and $C>0$ is some universal constant.

In KLS], it is conjectured that, 
Conjecture 1.1 There exists a universal constant $C$ such that $G_{n}<C$ for all $n \in \mathbb{N}$.

In this note we will show that, up to a small correction, the above is implied by a seemingly weaker hypothesis.

Next, we would like to formulate the thin-shell conjecture. Let $\sigma_{n} \geq 0$ satisfy

$$
\sigma_{n}^{2}=\sup _{X} \mathbb{E}\left[(|X|-\sqrt{n})^{2}\right]
$$

where the supremum runs over all isotropic, log-concave random vectors $X$ in $\mathbb{R}^{n}$. The shin-shell conjecture (see Anttila, Ball and Perissinaki [ABP and Bobkov and Koldobsky [BK] asserts the following:

Conjecture 1.2 There exists a universal constant $C$ such that,

$$
\sigma_{n}<C
$$

for all $n \in \mathbb{N}$.

An application of (3) with the function $\varphi(x)=|x|^{2}$ shows that the thin-shell conjecture is weaker than the KLS conjecture.

The first nontrivial bound for $\sigma_{n}$ was given by Klartag in [K1], who showed that $\sigma_{n} \leq C \frac{n^{1 / 2}}{\log (n+1)}$. Several improvements have been introduced around the same method, see e.g [K2] and [Fl1]. The best known bound for $\sigma_{n}$ at the time of this note is due to Guedon and E. Milman, in [Gu-M, extending previous works of Klartag, Fleury and Paouris, who show that $\sigma_{n} \leq C n^{\frac{1}{3}}$. The thin-shell conjecture was shown to be true for several specific classes of convex bodies, such as bodies with a symmetry for coordinate reflections (Klartag, K3]) and certain random bodies (Fleury, [F12]).

It was found by Sudakov, $\underline{\mathrm{Sud}}$, that the parameter $\sigma_{n}$ is highly related to almost-gaussian behaviour of certain marginals of a convex body, a fact now known as the central limit theorem for convex sets K1. This theorem asserts that most of the one-dimensional marginals of an isotropic, log-concave random vector are approximately gaussian in the sense that the Kolmogorov distance to the standard gaussian distribution of a typical marginal has roughly the order of magnitude of $\sigma_{n} / \sqrt{n}$. Therefore the conjectured bound (5) actually concerns the quality of the gaussian approximation to the marginals of high-dimensional log-concave measures.

The first theorem of this note reads,

Theorem 1.1 There exists a constant $C>0$ such that for all $n \geq 2$,

$$
G_{n} \leq C \sqrt{(\log n) \sum_{k=1}^{n} \frac{\sigma_{k}^{2}}{k}} .
$$


Note that, in particular, for any constant $\kappa>0$ such that $\sigma_{n} \leq n^{\kappa}$ for all $n \in \mathbb{N}$, one gets $G_{n} \leq C(\sqrt{\log n}) n^{\kappa}$. Under the thin-shell conjecture, the theorem gives $G_{n}<C \log n$.

Remark 1.3 Plugging the results of this paper into the currently best known bound for $\sigma_{n}$ (proven in $\left.[G u-M]\right), \sigma_{n} \leq C n^{1 / 3}$, it follows that

$$
G_{n} \leq C n^{1 / 3} \sqrt{\log n}
$$

This slightly improves the previous bound, $G_{n} \leq C n^{5 / 12}$, which is a corollary of $[G u-M]$ and $[B o]$.

Remark 1.4 In [EK1], B. Klartag and the author have found a connection between the thin-shell hypothesis and another well known conjecture related to convex bodies, known as the hyperplane conjecture. The methods of this paper share some common lines with the methods in [EK1]. In a very recent paper of K.Ball and V.H. Nguyen, [BN], a connection between the KLS conjecture and the hyperplane conjecture that applies for individual log-concave measures has also been established. They show that the isotropic constant of a log concave measure which attains a spectral gap is bounded by a constant which depends exponentially on the spectral gap.

Remark 1.5 Compare this result with the result in [Bo]. Bobkov's theorem states that for any log-concave random vector $X$ and any smooth function $\varphi$, one has

$$
\frac{\operatorname{Var}[\varphi(X)]}{\mathbb{E}\left[|\nabla \varphi(X)|^{2}\right]} \leq C \mathbb{E}[|X|] \sqrt{\operatorname{Var}[|X|]}
$$

Under the thin-shell hypothesis, Bobkov's theorem gives $G_{n} \leq C n^{1 / 4}$.

The bound in theorem 1.1 will rely on the following intermediate constant which corresponds to a slightly stronger thin shell bound. Define,

$$
K_{n}^{2}:=\sup _{X} \sup _{\theta \in S^{n-1}} \sum_{i, j=1}^{n} \mathbb{E}\left[X_{i} X_{j}\langle X, \theta\rangle\right]^{2},
$$

where the supremum runs over all isotropic log-concave random vectors $X$ in $\mathbb{R}^{n}$. Obviously, an equivalent definition of $K_{n}$ will be,

$$
K_{n}:=\sup _{\mu}\left\|\int_{\mathbb{R}^{n}} x_{1} x \otimes x d \mu(x)\right\|_{H S}
$$

where the supremum runs over all isotropic log-concave measures in $\mathbb{R}^{n}$. Here, $\|\cdot\|_{H S}$ stands for the Hilbert-Schmidt norm of a matrix.

There is a simple relation between $K_{n}$ and $\sigma_{n}$, namely,

Lemma 1.6 There exists a constant $C>0$ such that for all $n \geq 2$,

$$
K_{n} \leq C \sqrt{\sum_{k=1}^{n} \frac{\sigma_{k}^{2}}{k}} .
$$


Theorem 1.1 will be a consequence of the above lemma along with,

Proposition 1.7 There exists a constant $C>0$ such that for all $n \geq 2$,

$$
G_{n} \leq C K_{n} \sqrt{\log n} .
$$

Remark 1.8 The constant $K_{n}$ satisfies the following bound:

$$
K_{n}^{-1} \geq c \inf _{\mu} \inf _{E \subset \mathbb{R}^{n}} \frac{\mu^{+}(E)}{\mu(E)}
$$

where $\mu$ runs over all isotropic log-concave measures in $\mathbb{R}^{n}$, E runs over all ellipsoids with $\mu(E) \leq \frac{1}{2}$ and $c>0$ is some universal constant. This shows that up to the extra factor $\sqrt{\log n}$, in order to control the minimal possible surface area among all possible subsets of measure $\frac{1}{2}$ on the class of isotropic log-concave measures, it is enough to control the surface area of ellipsoids. See section 6 below for details.

We move on to the second result of this paper, a stability result for the Brunn-Minkowski Inequality. The Brunn-Minkowski inequality states, in one of its normalizations, that

$$
\operatorname{Vol}_{n}\left(\frac{K+T}{2}\right) \geq \sqrt{\operatorname{Vol}_{n}(K) \operatorname{Vol}_{n}(T)}
$$

for any compact sets $K, T \subset \mathbb{R}^{n}$, where $(K+T) / 2=\{(x+y) / 2 ; x \in$ $K, y \in T\}$ is half of the Minkowski sum of $K$ and $T$. When $K$ and $T$ are closed convex sets, equality in (7) holds if and only if $K$ is a translate of $T$.

When there is an almost-equality in (7), $K$ and $T$ are almost translates of each other in a certain sense (which varies between different estimates). Estimates of this form, often referred to as stability estimates, appear in Diskant Dis, in Groemer Groe, and in Figalli, Maggi and Pratelli FMP1, FMP2, Segal Seg.

The result [FMP2], which is essentially the strongest result in its category, and other existing stability estimates share a common thing: the bounds become worse as the dimension increases. In a recent paper, [EK2], Klartag and the author suggested that the correct bounds might actually become better as the dimension increases, as demonstrated by certain results. The estimates presented here may be viewed as a continuation of this line of research.

In order to formulate our result, we define the two constants

$$
\kappa=\liminf _{n \rightarrow \infty} \frac{\log \sigma_{n}}{\log n}, \quad \tau_{n}=\max \left(1, \max _{1 \leq j \leq n} \frac{\sigma_{j}}{j^{\kappa}}\right),
$$

so that $\sigma_{n} \leq \tau_{n} n^{\kappa}$. Note that the thin-shell conjecture implies $\kappa=0$ and $\tau_{n}<C$.

Our main estimate reads, 
Theorem 1.2 For every $\epsilon>0$ there exists a constant $C(\epsilon)$ such that the following holds: Let $K, T$ be convex bodies whose volume is 1 and whose barycenters lie at the origin. Suppose that the covariance matrix of the uniform measure on $K$ is equal to $L_{K} I d$ for a constant $L_{K}>0$. Denote,

$$
V=\operatorname{Vol}_{n}\left(\frac{K+T}{2}\right),
$$

and define

$$
\delta=C(\epsilon) L_{K} V^{5} \tau_{n} n^{2\left(\kappa-\kappa^{2}\right)+\epsilon}
$$

Then,

$$
\operatorname{Vol}_{n}\left(K_{\delta} \cap T\right) \geq 1-\epsilon
$$

Some remarks:

Remark 1.9 It follows from theorem 1.4 in [EK2] that the above estimate is true with

$$
\delta=C(\epsilon) \sqrt{\tau_{n}} n^{1 / 4+\kappa / 2} V^{5 / 2} .
$$

If $\kappa \geq 1 / 4$, then the result we prove here weaker than the one in [EK2]. However, under the thin shell hypothesis, the result of this paper becomes stronger, and is in fact tight up to the term $C(\epsilon) n^{\epsilon}$. This tightness is demonstrated, for instance, by taking $K$ and $T$ to be the unit cube and a unit cube truncated by a ball of radius $\sqrt{n}$ and normalized to be isotropic.

Remark 1.10 Using the bound in $[G u-M]$, the theorem gives

$$
\delta=C(\epsilon) n^{\frac{4}{9}+\epsilon} V^{5} L_{K} .
$$

Note that if the assumption (9) is dropped, even if the covariance matrices of $K$ and $T$ are assumed to be equal, the best corresponding bound would be $\delta=C \sqrt{n} L_{K}$ as demonstrated, for example, by a cube and a ball.

Remark 1.11 The above bound complements, in some sense, the result proven in [FMP1], which reads,

$$
\operatorname{Vol}_{n}\left(\left(K+x_{0}\right) \Delta T\right)^{2} \leq n^{7}\left(\operatorname{Vol}_{n}((K+T) / 2)-1\right)
$$

for some choice of $x_{0}$, where $\Delta$ denotes the symmetric difference between the sets. Unlike the result presented in this paper, the result in [FMP1] gives much more information as the expression $\operatorname{Vol}_{n}((K+T) / 2)-1$ approaches zero. On the other hand the result presented here already gives some information when $\operatorname{Vol}_{n}((K+T) / 2)=10$.

The structure of this paper is as follows: In section 2, we construct a stochastic localization scheme which will be the main ingredient our proofs. In section 3, we establish a bound for the covariance matrix of the measure throughout the localization process, which will be essential for its applications. In section 4, we prove theorem 1.1 and in section 5 we prove theorem 1.2 and its corollaries. In section 6 we tie some loose ends. 
Throughout this note, we use the letters $c, \tilde{c}, c^{\prime}, C, \tilde{C}, C^{\prime}, C^{\prime \prime}$ to denote positive universal constants, whose value is not necessarily the same in different appearances. Further notation used throughout the text: for a Borel measure $\mu$ on $\mathbb{R}^{n}, \operatorname{supp}(\mu)$ is the minimal closed set of full measure. The Euclidean unit ball is denoted by $B_{n}=\left\{x \in \mathbb{R}^{n} ;|x| \leq 1\right\}$. Its boundary is denoted by $S^{n-1}$. We write $\nabla \varphi$ for the gradient of the function $\varphi$, and $\nabla^{2} \varphi$ for the Hessian matrix. For a positive semi-definite symmetric matrix $A$, we denote its largest eigenvalue by $\|A\|_{O P}$. For any matrix $A$, we denote the sum of its diagonal entries by $\operatorname{Tr}(A)$, and by $\|A\|_{H S}^{2}$ we denote the sum of the eigenvalues of the matrix $A^{T} A$. For two densities $f, g$ on $\mathbb{R}^{n}$, define the Wasserstein distance, $W_{2}(f, g)$, by

$$
W_{2}(f, g)^{2}=\inf _{\xi} \int_{\mathbb{R}^{n} \times \mathbb{R}^{n}}|x-y|^{2} d \xi(x, y)
$$

where the infimum is taken over all measures $\xi$ on $\mathbb{R}^{2 n}$ whose marginals onto the first and last $n$ coordinates are the measures whose densities are $f$ and $g$ respectively (see, e.g. Vil] for more information).

Finally, for a continuous time stochastic process $X_{t}$, we denote by $d X_{t}$ the differential of $X_{t}$, and by $[X]_{t}$ the quadratic variation of $X_{t}$. For a pair of continuous time stochastic processes $X_{t}, Y_{t}$, the quadratic covariation will be denoted by $[X, Y]_{t}$.

Acknowledgements I owe this work to countless useful discussions I have had with my supervisor, Bo'az Klartag, through which I learnt the vast part of what I know about the subject, as well as about related topics, and for which I am grateful. I would also like to thank Vitali and Emanuel Milman and Boris Tsirelson for inspiring discussions and for their useful remarks on a preliminary version of this note. Finally, I would like to thank the anonymous referee for doing a tremendous job reviewing a preliminary version of this paper, thanks to his/her ideas the proofs are significantly simpler, shorter and more comprehensible.

\section{A stochastic localization scheme}

In this section we construct the localization scheme which will be the principal component in our proofs. The construction will use elementary properties of semimartingales and stochastic integration. For definitions, see Dur.

For the construction, we assume that we are given some isotropic random vector $X \in \mathbb{R}^{n}$ with density $f(x)$. Well-known concentration bounds for log-concave measures (see, e.g., section 2 of $[\mathrm{K} 2$ ) will allow us to assume throughout the paper that

$$
\operatorname{supp}(f) \subseteq n B_{n},
$$

where $B_{n}$ is the Euclidean ball of radius 1 . 
We begin with some definitions. For a vector $c \in \mathbb{R}^{n}$ and an $n \times n$ matrix $B$, we write

$$
V_{f}(c, B)=\int_{\mathbb{R}^{n}} e^{\langle c, x\rangle-\frac{1}{2}\langle B x, x\rangle} f(x) d x .
$$

Define a vector valued function,

$$
a_{f}(c, B)=V_{f}^{-1}(c, B) \int_{\mathbb{R}^{n}} x e^{\langle c, x\rangle-\frac{1}{2}\langle B x, x\rangle} f(x) d x,
$$

and a matrix valued function,

$A_{f}(c, B)=V_{f}^{-1}(c, B) \int_{\mathbb{R}^{n}}\left(x-a_{f}(c, B)\right) \otimes\left(x-a_{f}(c, B)\right) e^{\langle c, x\rangle-\frac{1}{2}\langle B x, x\rangle} f(x) d x$.

The assumption (10) ensures that $V_{f}, a_{f}$ and $A_{f}$ are smooth functions of $c, B$.

Let $W_{t}$ be a standard Wiener process and consider the following system of stochastic differential equations:

$$
\begin{gathered}
c_{0}=0, \quad d c_{t}=A_{f}^{-1 / 2}\left(c_{t}, B_{t}\right) d W_{t}+A_{f}^{-1}\left(c_{t}, B_{t}\right) a_{f}\left(c_{t}, B_{t}\right) d t \\
B_{0}=0, \quad d B_{t}=A_{f}^{-1}\left(c_{t}, B_{t}\right) d t .
\end{gathered}
$$

Taking into account the fact that the functions $A_{f}, a_{f}$ are smooth and that $A_{f}(c, B)$ is positive definite for all $c, B$, we can use a standard existence and uniqueness theorem (see e.g., $\mathrm{Ok}$, section 5.2) to ensure the existence and uniqueness of a solution in some interval $0 \leq t \leq t_{0}$, where $t_{0}$ is an almost-surely positive random variable.

Next, we construct a 1-parameter family of functions $\Gamma_{t}(f)$ by defining,

$$
F_{t}(x)=V_{f}^{-1}\left(c_{t}, B_{t}\right) e^{\left\langle c_{t}, x\right\rangle-\frac{1}{2}\left\langle B_{t} x, x\right\rangle}
$$

and

$$
\Gamma_{t}(f)(x)=f(x) F_{t}(x)
$$

Also, abbreviate

$$
a_{t}=a_{f}\left(c_{t}, B_{t}\right), \quad A_{t}=A_{f}\left(c_{t}, B_{t}\right), \quad V_{t}=V_{f}\left(c_{t}, B_{t}\right), \quad f_{t}=\Gamma_{t}(f),
$$

so that $a_{t}$ and $A_{t}$ are the barycenter and the covariance matrix of the function $f_{t}$.

The following lemma may shed some light on this construction.

Lemma 2.1 The function $F_{t}$ satisfies the following set of equations:

$$
\begin{gathered}
F_{0}(x)=1, \quad d F_{t}(x)=\left\langle x-a_{t}, A_{t}^{-1 / 2} d W_{t}\right\rangle F_{t}(x), \\
a_{t}=\int_{\mathbb{R}^{n}} x f(x) F_{t}(x) d x, \quad A_{t}=\int_{\mathbb{R}^{n}}\left(x-a_{t}\right) \otimes\left(x-a_{t}\right) f(x) F_{t}(x) d x,
\end{gathered}
$$

for all $x \in \mathbb{R}^{n}$ and all $0 \leq t \leq t_{0}$. 
Proof:

Fix $x \in \mathbb{R}^{n}$. We will show that $d F_{t}(x)=\left\langle x-a_{t}, A_{t}^{-1 / 2} d W_{t}\right\rangle F_{t}(x)$. The correctness of the other equations is obvious. Define,

$$
G_{t}(x)=V_{t} F_{t}(x)=e^{\left\langle c_{t}, x\right\rangle-\frac{1}{2}\left\langle B_{t} x, x\right\rangle} .
$$

Equation (11) clearly implies that $[B]_{t}=0$. Let $Q_{t}(x)$ denote the quadratic variation of the process $\left\langle x, c_{t}\right\rangle$. We have,

$$
d\left\langle x, c_{t}\right\rangle=\left\langle A_{t}^{-1 / 2} x, d W_{t}+A_{t}^{-1 / 2} a_{t} d t\right\rangle
$$

It follows that,

$$
d Q_{t}(x)=\left\langle A_{t}^{-1} x, x\right\rangle d t .
$$

Using Itô's formula, we calculate

$$
\begin{gathered}
d G_{t}(x)=\left(\left\langle x, d c_{t}\right\rangle-\frac{1}{2}\left\langle d B_{t} x, x\right\rangle+\frac{1}{2} d Q_{t}(x)\right) G_{t}(x)= \\
\left(\left\langle x, A_{t}^{-1 / 2} d W_{t}+A_{t}^{-1} a_{t} d t\right\rangle-\frac{1}{2}\left\langle A_{t}^{-1} x, x\right\rangle d t+\frac{1}{2}\left\langle A_{t}^{-1} x, x\right\rangle d t\right) G_{t}(x)= \\
\left\langle x, A_{t}^{-1 / 2} d W_{t}+A_{t}^{-1} a_{t} d t\right\rangle G_{t}(x)
\end{gathered}
$$

Next, we calculate,

$$
\begin{gathered}
d V_{t}(x)=d \int_{\mathbb{R}^{n}} e^{\left\langle c_{t}, x\right\rangle-\frac{1}{2}\left\langle B_{t} x, x\right\rangle} f(x) d x= \\
\int_{\mathbb{R}^{n}} d G_{t}(x) f(x) d x=\int_{\mathbb{R}^{n}}\left\langle x, A_{t}^{-1 / 2} d W_{t}+A_{t}^{-1} a_{t} d t\right\rangle G_{t}(x) f(x) d x= \\
V_{t}\left\langle a_{t}, A_{t}^{-1 / 2} d W_{t}+A_{t}^{-1} a_{t} d t\right\rangle .
\end{gathered}
$$

So, using Itô's formula again,

$$
\begin{gathered}
d V_{t}^{-1}=-\frac{d V_{t}}{V_{t}^{2}}+\frac{d[V]_{t}}{V_{t}^{3}}= \\
-V_{t}^{-1}\left\langle a_{t}, A_{t}^{-1 / 2} d W_{t}+A_{t}^{-1} a_{t} d t\right\rangle+V_{t}^{-1}\left\langle A_{t}^{-1} a_{t}, a_{t}\right\rangle .
\end{gathered}
$$

Applying Itô's formula one last time yields,

$$
\begin{gathered}
d F_{t}(x)=d\left(V_{t}^{-1} G_{t}(x)\right)= \\
G_{t}(x) d V_{t}^{-1}+V_{t}^{-1} d G_{t}(x)+d\left[V^{-1}, G(x)\right]_{t}= \\
-V_{t}^{-1}\left\langle a_{t}, A_{t}^{-1 / 2} d W_{t}+A_{t}^{-1} a_{t} d t\right\rangle G_{t}(x)+V_{t}^{-1}\left\langle A_{t}^{-1} a_{t}, a_{t}\right\rangle G_{t}(x)+ \\
+V_{t}^{-1}\left\langle x, A_{t}^{-1 / 2} d W_{t}+A_{t}^{-1} a_{t} d t\right\rangle G_{t}(x)-\left\langle A_{t}^{-1 / 2} a_{t}, A_{t}^{-1 / 2} x\right\rangle V_{t}^{-1} G_{t}(x) d t= \\
\left\langle A_{t}^{-1 / 2} d W_{t}, x-a_{t}\right\rangle F_{t}(x) .
\end{gathered}
$$

This finishes the proof. 
Remark 2.2 In view of the above lemma it can be seen that, in some sense, the above is just the continuous version of the following iterative process: at every time step, multiply the function by a linear function equal to 1 at the barycenter, whose gradient has a random direction distributed uniformly on the ellipsoid of inertia. This construction may also be thought of as a variant of the Brownian motion on the Riemannian manifold constructed in [EK1].

Remark 2.3 Rather than defining the process $F_{t}$ through equations (11) and (12), one may alternatively define it directly with the infinite system of stochastic differential equations in formula (13). In this case, the existence and uniqueness of the solution can be shown using [KX], Theorem 5.2.2, page 159] (however, some extra work is needed in order to show that the conditions of this theorem hold).

In the remainder of this note, most of the calculations involving the process $f_{t}$ will use the formula (13) rather than the formulas (11) and (12).

The remaining part of this section is dedicated to analyzing some basic properties of $\Gamma_{t}(f)$. We begin with:

Lemma 2.4 The process $\Gamma_{t}(f)$ satisfies the following properties:

(i) The function $\Gamma_{t}(f)$ is almost surely well defined, finite and log-concave for all $t>0$.

(ii) For all $t>0, \int_{\mathbb{R}^{n}} f_{t}(x) d x=1$.

(iii) The process has a semi-group property, namely,

$$
\Gamma_{s+t}(f) \sim \frac{1}{\sqrt{\operatorname{det} A_{s}}} \Gamma_{t}\left(\sqrt{\operatorname{det} A_{s}} \Gamma_{s}(f) \circ L^{-1}\right) \circ L,
$$

where

$$
L(x)=A_{s}^{-1 / 2}\left(x-a_{s}\right) .
$$

(iv) For every $x \in \mathbb{R}^{n}$, the process $f_{t}(x)$ is a martingale.

In order to prove (i), we will first need the following technical lemma:

Lemma 2.5 For every dimension $n$, there exists a constant $c(n)>0$ such that,

$$
\mathbb{P}\left(A_{t} \geq c(n) I d, \quad \forall 0 \leq t \leq c(n)\right) \geq c(n) .
$$

The proof of this lemma is postponed section 6 .

Proof of lemma 2.4.

To prove (i), we have to make sure that $A_{t}^{-1 / 2}$ does not blow up. To this end, define $t_{0}=\inf \left\{t \mid \operatorname{det} A_{t}=0\right\}$. By continuity, $t_{0}>0$. Equation (12) suggests that $f_{t}$ is log-concave for all $t<t_{0}$. The fact that $t_{0}=\infty$ will be proven below.

We start by showing that both (ii) and (iii) hold for any $t<t_{0}$.

We first calculate, using (13),

$$
d \int_{\mathbb{R}^{n}} f(x) F_{t}(x) d x=\int_{\mathbb{R}^{n}} f(x) d F_{t}(x) d x=
$$




$$
\int_{\mathbb{R}^{n}} f(x) F_{t}(x)\left\langle A_{t}^{-1 / 2} d W_{t}, x-a_{t}\right\rangle d x=0,
$$

with probability 1 . The last equality follows from the definition of $a_{t}$ as the barycenter of the measure $f(x) F_{t}(x) d x$. We conclude (ii).

We continue with proving (iii). To do this, fix some $0<s<t_{0}-t$ and write,

$$
L(x)=A_{s}^{-1 / 2}\left(x-a_{s}\right) .
$$

We normalize $f_{s}$ by defining,

$$
g(x)=\sqrt{\operatorname{det} A_{s}} f_{s}\left(L^{-1}(x)\right),
$$

which is clearly an isotropic probability density. Let us inspect $\Gamma_{t}(g(x))$. We have, using (13),

$$
\begin{gathered}
\left.d \Gamma_{t}(g)(x)\right|_{t=0}=g(x)\left\langle x, d W_{t}\right\rangle=\sqrt{\operatorname{det} A_{s}} f_{s}\left(L^{-1}(x)\right)\left\langle L\left(L^{-1}(x)\right), d W_{t}\right\rangle= \\
\sqrt{\operatorname{det} A_{s}} f_{s}\left(L^{-1}(x)\right)\left\langle L^{-1}(x)-a_{s}, A_{s}^{-1 / 2} d W_{t}\right\rangle,
\end{gathered}
$$

On the other hand,

$$
d f_{s}\left(L^{-1}(x)\right)=f_{s}\left(L^{-1}(x)\right)\left\langle L^{-1}(x)-a_{s}, A_{s}^{-1 / 2} d W_{s}\right\rangle
$$

in other words,

$$
\left.\left.d \Gamma_{t}\left(\sqrt{\operatorname{det} A_{s}} \Gamma_{s}(f) \circ L^{-1}\right)\right|_{t=0} \sim \sqrt{\operatorname{det} A_{s}} d \Gamma_{t}(f) \circ L^{-1}\right|_{t=s}
$$

which proves (iii).

We are left with showing that $t_{0}=\infty$. To see this, write,

$$
s_{1}=\min \left\{t \quad ; \quad\left\|A_{t}^{-1}\right\|_{O P}=c^{-1}(n)\right\},
$$

where $c(n)$ is the constant from lemma 2.5 Note that, by continuity, $s_{1}$ is well-defined and almost-surely positive. When time $s$ comes, we may define $L_{1}$ as in (15), and continue running the process on the function $f \circ L_{1}^{-1}$ as above. We repeat this every time $\left\|A_{t}^{-1}\right\|_{O P}$ hits the value $c^{-1}(n)$, thus generating the hitting times $s_{1}, s_{2}, \ldots$ Lemma 2.5 suggests that,

$$
\mathbb{P}\left(s_{i+1}-s_{i}>c(n) \mid s_{1}, s_{2}, \ldots, s_{i}\right)>c(n),
$$

which implies that, almost surely, $s_{i+1}-s_{i}>c(n)$ for infinitely many values of $i$. Thus, $\lim _{n \rightarrow \infty} s_{n}=\infty$ almost surely, and so $t_{0}=+\infty$.

Part (iv) follows immediately from formula (13). The lemma is proven.

Our next task is to analyze the path of the barycenter $a_{t}=\int_{\mathbb{R}^{n}} x f_{t}(x) d x$. We have, using (13),

$$
\begin{gathered}
d a_{t}=d \int_{\mathbb{R}^{n}} x f(x) F_{t}(x) d x=\int_{\mathbb{R}^{n}} x f(x) F_{t}(x)\left\langle x-a_{t}, A_{t}^{-1 / 2} d W_{t}\right\rangle d x= \\
\left(\int_{\mathbb{R}^{n}}\left(x-a_{t}\right) \otimes\left(x-a_{t}\right) f_{t}(x) d x\right)\left(A_{t}^{-1 / 2} d W_{t}\right)=A_{t}^{1 / 2} d W_{t} .
\end{gathered}
$$


where the third equality follows from the defition of $a_{t}$, which implies,

$$
\int_{\mathbb{R}^{n}} a_{t} f(x) F_{t}(x)\left\langle x-a_{t}, A_{t}^{-1 / 2} d W_{t}\right\rangle=0 .
$$

One of the crucial points, when using this localization scheme, will be to show that the barycenter of the measure does not move too much throughout the process. For this, we would like to attain upper bounds on the eigenvalues of the matrix $A_{t}$. We start with a simple observation:

Equation (12) shows that the measure $f_{t}$ is log-concave with respect to the measure $e^{-\frac{1}{2}\left|B_{t}^{1 / 2} x\right|^{2}}$. The following result, which is well-known to experts, shows that measures which possess this property attain certain concentration inequalities.

Proposition 2.6 There exists a universal constant $\Theta>0$ such that the following holds: Let $\phi: \mathbb{R}^{n} \rightarrow \mathbb{R}$ be a convex function and let $K>0$. Suppose that,

$$
d \mu(x)=Z e^{-\phi(x)-\frac{1}{2 K^{2}}|x|^{2}} d x
$$

is a probability measure whose barycenter lies at the origin. Then,

(i) For all Borel sets $A \subset \mathbb{R}^{n}$, with $0.1 \leq \mu(A) \leq 0.9$, one has,

$$
\mu\left(A_{K \Theta}\right) \geq 0.95
$$

where $A_{K \Theta}$ is the $K \Theta$-extension of $A$, defined in the previous section.

(ii) For all $\theta \in S^{n-1}$,

$$
\int\langle x, \theta\rangle^{2} d \mu(x) \leq \Theta K^{2}
$$

Proof:

Denote the density of $\mu$ by $\rho(x)$. Let $B$ be the complement of $A_{K \Theta}$, where the constant $\Theta$ will be chosen later on. Define,

$$
f(x)=\rho(x) \mathbf{1}_{A}, \quad g(x)=\rho(x) \mathbf{1}_{B} .
$$

Note that for $x \in A$ and $y \in B$, we have $|x-y|>K \Theta$. Thus, by the parallelogram law,

$$
\left|\frac{x+y}{2}\right|^{2} \leq \frac{|x|^{2}+|y|^{2}}{2}-\frac{1}{4} K^{2} \Theta^{2}
$$

which implies,

$$
e^{-\frac{1}{2 K^{2}}\left|\frac{x+y}{2}\right|^{2}} \geq \sqrt{e^{-\frac{1}{2 K^{2}}|x|^{2}} e^{-\frac{1}{2 K^{2}}|x|^{2}}} e^{\frac{1}{8} \Theta^{2}} .
$$

Since the function $\phi$ is assumed to be convex, we obtain

$$
\rho\left(\frac{x+y}{2}\right) \geq \sqrt{f(x) g(y)} e^{\frac{1}{8} \Theta^{2}} .
$$

Now, using the Prekopa-Leindler theorem, we attain

$$
\mu(A) \mu(B)=\int_{\mathbb{R}^{n}} f(x) d x \int_{\mathbb{R}^{n}} g(x) d x \leq e^{-\frac{1}{4} \Theta^{2}},
$$


so,

$$
\mu\left(A_{K \Theta}\right) \geq 1-\frac{e^{-\frac{1}{4} \Theta^{2}}}{\mu(A)} \geq 1-10 e^{-\frac{1}{4} \Theta^{2}} .
$$

Clearly, a large enough choice of the constant $\Theta$ gives (i). To prove (ii), we define,

$$
g(t)=\mu(\{x ;\langle x, \theta\rangle \geq t\}),
$$

and take $A=\left\{x ;\langle x, \theta\rangle<g^{-1}(0.5)\right\}$. An application on (i) on the set $A$ gives,

$$
g\left(g^{-1}(0.5)+K \Theta\right) \leq 0.05
$$

since $g$ is log-concave, we attain

$$
g\left(g^{-1}(0.5)+t K \Theta\right) \leq 0.05^{t}, \quad \forall t>1
$$

and in the same way, one can attain,

$$
1-g\left(g^{-1}(0.5)-t K \Theta\right) \leq 0.05^{t}, \quad \forall t>1 .
$$

Part (ii) of the proposition is a direct consequence of the last two equations.

Plugging (12) into part (ii) of this theorem gives,

$$
A_{t} \leq \Theta\left\|B_{t}^{-1}\right\|_{O P} I d \leq \Theta\left(\int_{0}^{t} \frac{d s}{\left\|A_{s}\right\|_{O P}}\right)^{-1} I d, \quad \forall t>0 .
$$

By our assumption (10) we deduce that $A_{t}$ is bounded by $n^{2} I d$, which immediately gives

$$
A_{t}<\frac{\Theta n^{2}}{t} I d
$$

The bound (18) will be far from sufficient for our needs, and the next section is dedicated to attaining a better upper bound. However, it is good enough to show that the barycenter, $a_{t}$, converges in distribution to the density $f(x)$.

Indeed, (18) implies that

$$
\lim _{t \rightarrow \infty} W_{2}\left(f_{t}, \delta_{a_{t}}\right)=0
$$

where $\delta_{a_{t}}$ is the probability measure supported on $\left\{a_{t}\right\}$. In other words the probability density $f_{t}(x)$ converges to a delta measure. By the martingale property, part (iv) of lemma 2.4 we know that $\mathbb{E}\left[f_{t}(x)\right]=f(x)$, thus, $X_{t}:=a_{t}$ converges, in Wasserstein metric, to the original random vector $X$ as $t \rightarrow \infty$.

Remark 2.7 It is interesting to compare this construction with the construction by Lehec in [Leh]. In both cases, a certain Itô process converges to a given log-concave measure. In the result of Lehec, the convergence is ensured by applying a certain adapted drift, while here, it is ensured by adjusting the covariance matrix of the process. 
We end this section with a simple calculation in which we analyze the process $\Gamma_{t}(f)$ in the simple case that $f$ is the standard Gaussian measure. While the calculation will not be necessary for our proofs, it may provide the reader a better understanding of the process. Define,

$$
f(x)=(2 \pi)^{-n / 2} e^{-|x|^{2} / 2} .
$$

According to formula (12), the function $f_{t}$ takes the form,

$$
f_{t}(x)=V_{t}^{-1} \exp \left(\left\langle x, c_{t}\right\rangle-\frac{1}{2}\left\langle\left(B_{t}+I d\right) x, x\right\rangle\right)
$$

where $V_{t} \in R, c_{t} \in \mathbb{R}^{n}$ are certain Itô processes. It follows that the covariance matrix $A_{t}$ satisfies,

$$
A_{t}^{-1}=B_{t}+I d .
$$

Recall that $B_{t}=\int_{0}^{t} A_{s}^{-1} d s$. It follows that,

$$
\frac{d}{d t} B_{t}=B_{t}+I d, \quad B_{0}=0
$$

So,

$$
B_{t}=\left(e^{t}-1\right) I d
$$

which gives,

$$
A_{t}=e^{-t} I d
$$

Next, we use (16) to derive that,

$$
d a_{t}=e^{-t / 2} d W_{t}
$$

which implies,

$$
a_{t} \sim W_{1-\exp (-t)}
$$

We finally get,

$$
f_{t}=e^{n t / 2}(2 \pi)^{-n / 2} \exp \left(-\frac{1}{2} e^{t}\left|\left(x-W_{1-\exp (-t)}\right)\right|^{2}\right) .
$$

\section{Analysis of the matrix $A_{t}$}

In the previous section we saw that the covariance matrix of the $f_{t}, A_{t}$, satisfies (18). The goal of this section is to give a better bound, which holds also for small $t$. Namely, we want to prove:

Proposition 3.1 There exist universal constants $C, c>0$ such that for all $n \geq 2$ the following holds: Let $f: \mathbb{R}^{n} \rightarrow \mathbb{R}_{+}$be an isotropic, log concave probability density. Let $A_{t}$ be the covariance matrix of $\Gamma_{t}(f)$. Then, (i) Define the event $F$ by,

$$
F:=\left\{\left\|A_{t}\right\|_{O P}<C K_{n}^{2}(\log n) e^{-c t}, \quad \forall t>0\right\} .
$$

One has,

$$
\mathbb{P}(F) \geq 1-\left(n^{-10}\right)
$$


(ii) For all $t>0, \mathbb{E}\left[\operatorname{Tr}\left(A_{t}\right)\right] \leq n$.

(iii) Whenever the event $F$ holds, the following also holds:

For all $t>\frac{1}{K_{n}^{2} \log n}$ there exists a convex function $\phi_{t}(x)$ such that the function $f_{t}$ is of the form,

$$
f_{t}(x)=\exp \left(-\left|\frac{x}{C K_{n} \sqrt{\log n}}\right|^{2}-\phi_{t}(x)\right) .
$$

Before we move on to the proof, we will establish some simple properties of the matrix $A_{t}$. Our first task is to find the differential of process $A_{t}$. We have, using Itô's formula with equation (13),

$$
\begin{gathered}
d A_{t}=d \int_{\mathbb{R}^{n}}\left(x-a_{t}\right) \otimes\left(x-a_{t}\right) f_{t}(x) d x= \\
\int_{\mathbb{R}^{n}}\left(x-a_{t}\right) \otimes\left(x-a_{t}\right) d f_{t}(x) d x-2 \int_{\mathbb{R}^{n}} d a_{t} \otimes\left(x-a_{t}\right) f_{t}(x) d x- \\
-2 \int_{\mathbb{R}^{n}}\left(x-a_{t}\right) \otimes d\left[a_{t}, f_{t}(x)\right]_{t} d x+d\left[a_{t}, a_{t}\right] \int_{\mathbb{R}^{n}} f_{t}(x) d x .
\end{gathered}
$$

Let us try to understand each of this terms. The second term is,

$$
\int_{\mathbb{R}^{n}} d a_{t} \otimes\left(x-a_{t}\right) f_{t}(x) d x=d a_{t} \otimes \int_{\mathbb{R}^{n}}\left(x-a_{t}\right) f_{t} d t=0 .
$$

Recall that by (16), $d a_{t}=A_{t}^{1 / 2} d W_{t}$, which gives,

$$
d\left[a_{t}, a_{t}\right]_{t}=A_{t} d t
$$

and

$$
d\left[a_{t}, f_{t}(x)\right]=f_{t}(x) A_{t}^{1 / 2} A_{t}^{-1 / 2} x d t=f_{t}(x) x d t,
$$

which implies,

$$
\begin{gathered}
\int_{\mathbb{R}^{n}}\left(x-a_{t}\right) \otimes d\left[a_{t}, f_{t}(x)\right]_{t} d x=\int_{\mathbb{R}^{n}}\left(x-a_{t}\right) \otimes x f_{t}(x) d x d t= \\
\int_{\mathbb{R}^{n}}\left(x-a_{t}\right) \otimes\left(x-a_{t}\right) f_{t}(x) d x d t=A_{t} d t
\end{gathered}
$$

Plugging equations (23), (24) and (25) together gives,

$$
d A_{t}=\int_{\mathbb{R}^{n}}\left(x-a_{t}\right) \otimes\left(x-a_{t}\right) d f_{t}(x) d x-A_{t} d t
$$

so we finally get,

$$
d A_{t}=\int_{\mathbb{R}^{n}}\left(x-a_{t}\right) \otimes\left(x-a_{t}\right)\left\langle x-a_{t}, A_{t}^{-1 / 2} d W_{t}\right\rangle f_{t}(x) d x-A_{t} d t .
$$

Note that the term $A_{t} d t$ is positive definite, hence, the fact it appears in the differential can only make all of the eigenvalues of $A_{t}$ smaller (as a matter of fact, this term induces a rather strong drift of all the eigenvalues 
towards 0 , which we will not even use). Consequently, we can define $\tilde{A}_{t}=A_{t}+\int_{0}^{t} A_{s} d s$, so that

$$
d \tilde{A}_{t}=\int_{\mathbb{R}^{n}}\left(x-a_{t}\right) \otimes\left(x-a_{t}\right)\left\langle x-a_{t}, A_{t}^{-1 / 2} d W_{t}\right\rangle f_{t}(x) d x
$$

and $\tilde{A}_{0}=A_{0}=I d$. Clearly, $A_{t} \leq \tilde{A}_{t}$ for all $t>0$. In order to control $\left\|A_{t}\right\|_{O P}$, it is thus enough to bound $\left\|\tilde{A}_{t}\right\|_{O P}$.

For a fixed value of $t$, let $v_{1}, \ldots, v_{n}$ be an orthonormal basis, with respect to which $\tilde{A}_{t}$ is diagonal, and write $\alpha_{i, j}=\left\langle v_{i}, \tilde{A}_{t} v_{j}\right\rangle$ for the entries of $\tilde{A}_{t}$ with respect to this basis. Equation (26) can be written,

$$
d \alpha_{i, j}=\int_{\mathbb{R}^{n}}\left\langle x, v_{i}\right\rangle\left\langle x, v_{j}\right\rangle\left\langle A_{t}^{-1 / 2} x, d W_{t}\right\rangle f_{t}\left(x+a_{t}\right) d x
$$

Next, denote

$$
\xi_{i, j}=\frac{1}{\sqrt{\alpha_{i, i} \alpha_{j, j}}} \int_{\mathbb{R}^{n}}\left\langle x, v_{i}\right\rangle\left\langle x, v_{j}\right\rangle A_{t}^{-1 / 2} x f_{t}\left(x+a_{t}\right) d x .
$$

So,

$$
d \alpha_{i, j}=\sqrt{\alpha_{i, i} \alpha_{j, j}}\left\langle\xi_{i, j}, d W_{t}\right\rangle
$$

and

$$
\frac{d}{d t}\left[\alpha_{i, j}\right]_{t}=\alpha_{i, i} \alpha_{j, j}\left|\xi_{i, j}\right|^{2}
$$

As we will witness later, behaviour norm of the matrix $\tilde{A}_{t}$ depends highly on the norms of the vectors $\xi_{i, j}$, which induce a certain repulsion between the eigenvalues. The next lemma will come in handy when we need to bound these norms:

Lemma 3.2 The vectors $\xi_{i, j}$ satisfy the following bounds:

(i) For all $1 \leq i \leq n,\left|\xi_{i, i}\right|<C$ for some universal constant $C>0$.

(ii) For all $1 \leq i \leq n, \sum_{j=1}^{n}\left|\xi_{i, j}\right|^{2} \leq K_{n}^{2}$.

Proof:

Since $A_{t}^{1 / 2} v_{i}=\sqrt{\alpha_{i, i}} v_{i}$ for all $1 \leq i \leq n$, we have

$$
\xi_{i, j}=\int_{\mathbb{R}^{n}}\left\langle A_{t}^{-1 / 2} x, v_{i}\right\rangle\left\langle A_{t}^{-1 / 2} x, v_{j}\right\rangle A_{t}^{-1 / 2} x f_{t}\left(x+a_{t}\right) d x
$$

Define again, as above, $\tilde{f}_{t}(x)=\sqrt{\operatorname{det} A_{t}} f_{t}\left(A_{t}^{1 / 2} x+a_{t}\right)$. By substituting $y=A_{t}^{-1 / 2} x$, the equation becomes

$$
\xi_{i, j}=\int_{\mathbb{R}^{n}}\left\langle y, v_{i}\right\rangle\left\langle y, v_{j}\right\rangle y \tilde{f}_{t}(y) d y .
$$

Recall that $\tilde{f}_{t}$ is isotropic. The last equation shows that the vectors $\xi_{i, j}$, in some sense, do not depend on the position of $f$. Using the CauchySchwartz inequality, one has

$$
\left|\xi_{i, i}\right|=\left|\int_{\mathbb{R}^{n}}\left\langle y, v_{i}\right\rangle^{2} y \tilde{f}_{t}(y) d y\right|=\left|\int_{\mathbb{R}^{n}}\left\langle y, v_{i}\right\rangle^{2}\left\langle y, \frac{\xi_{i, i}}{\left|\xi_{i, i}\right|}\right\rangle \tilde{f}_{t}(y) d y\right| \leq
$$




$$
\sqrt{\int_{\mathbb{R}^{n}}\left\langle y, v_{i}\right\rangle^{4} \tilde{f}_{t}(y) d y \int_{\mathbb{R}^{n}}\left\langle y, \frac{\xi_{i, i}}{\left|\xi_{i, i}\right|}\right\rangle^{2} \tilde{f}_{t}(y) d y} .
$$

A well-known fact about isotropic log-concave measures (see for example LV] Lemma 5.7]) is that for every $p>0$ there exists a constant $c(p)$ such that for every isotropic log-concave density $\rho(x)$ on $\mathbb{R}^{n}$ and every $\theta \in S^{n-1}$,

$$
\int_{\mathbb{R}^{n}}|\langle x, \theta\rangle|^{p} \rho(x) d x \leq c(p) .
$$

Using this with (30) establishes (i). Next, by the definition of $K_{n}$, we have for all $1 \leq i \leq n$,

$$
\begin{aligned}
\sum_{j=1}^{n}\left|\xi_{i, j}\right|^{2} & =\sum_{j=1}^{n} \sum_{k=1}^{n}\left|\int_{\mathbb{R}^{n}}\left\langle y, v_{i}\right\rangle\left\langle y, v_{j}\right\rangle\left\langle y, v_{k}\right\rangle \tilde{f}_{t}(y) d y\right|^{2}= \\
& \left\|\int_{\mathbb{R}^{n}} y \otimes y\left\langle y, v_{i}\right\rangle \tilde{f}_{t}(y) d y\right\|_{H S}^{2} \leq K_{n}^{2} .
\end{aligned}
$$

The lemma is proven.

We are now ready to prove the main proposition of the section.

Proof of proposition 3.1

We fix a positive integer $p$ whose value will be chosen later, and define,

$$
S_{t}=\operatorname{Tr}\left(\tilde{A}_{t}^{p}\right)
$$

Since $S_{t}$ is a smooth function of the coefficients $\left\{\alpha_{i, j}\right\}$, which are Itô processes (assuming that the basis $v_{1}, \ldots, v_{n}$ is fixed), $S_{t}$ itself is also an Itô process. Fix some $t>0$. Our next goal will be to find $d S_{t}$. To that end, define $\Gamma$ to be the set of $(p+1)$-tuples, $\left(j_{1}, \ldots, j_{p+1}\right)$, such that $j_{i} \in\{1, \ldots, n\}$ for all $1 \leq i \leq p+1$ and such that $j_{1}=j_{p+1}$. It is easy to verify that,

$$
S_{t}=\sum_{\left(j_{1}, \ldots, j_{p+1}\right) \in \Gamma} \alpha_{j_{1}, j_{2}} \alpha_{j_{2}, j_{3}} \cdots \alpha_{j_{p}, j_{p+1}} .
$$

Since $\operatorname{Tr}\left(\tilde{A}_{t}^{p}\right)$ does not depend on the choice of orthogonal coordinates, after fixing the value of $t$, we are free to choose our coordinates such that the matrix $\tilde{A}_{t}$ is diagonal, thus assuming that $\alpha_{i, j}=0$ whenever $i \neq j$ and that (28) holds (in other words, we calculate the differential $d S_{t}$ using a basis $v_{1}, \ldots, v_{n}$ which depends on $t$. However, after fixing the value of $t$, the calculation itself is with respect to a fixed basis). A moment of reflection reveals that, in this case, the term $d\left(\alpha_{j_{1}, j_{2}} \cdots \alpha_{j_{p}, j_{p+1}}\right)$ can be non-zero only if there are at most two distinct indices $i_{1}, i_{2}$ such that $j_{i_{1}} \neq j_{i_{1}+1}$ and $j_{i_{2}} \neq j_{i_{2}+1}$. We are left with two types of terms whose differential is non-zero. The first type of term contains no off-diagonal entries, and has the form $\left(\alpha_{i, i}\right)^{p}$. Using equation (28), we calculate its differential,

$$
d \alpha_{i, i}^{p}=p \alpha_{i, i}^{p-1} d \alpha_{i, i}+\frac{1}{2} p(p-1) \alpha_{i, i}^{p-2} d\left[\alpha_{i, j}\right]_{t}=
$$




$$
p \alpha_{i, i}^{p}\left\langle\xi_{i, i}, d W_{t}\right\rangle+p(p-1) \alpha_{i, i}^{p}\left|\xi_{i, i}\right|^{2} d t .
$$

The second type of term will contain exactly two off-diagonal entries, and due to the symmetry of the matrix and the constraint $j_{1}=j_{p+1}$, it has the form:

$$
\left(\alpha_{i, i}\right)^{k_{1}} \alpha_{i, j}\left(\alpha_{j, j}\right)^{k_{2}} \alpha_{j, i}\left(\alpha_{i, i}\right)^{k_{3}}=\left(\alpha_{i, i}\right)^{k}\left(\alpha_{j, j}\right)^{p-k-2}\left(\alpha_{i, j}\right)^{2}
$$

where $i \neq j$ and $0 \leq k \leq p-2$. Keeping in mind that $\alpha_{i, j}=0$, we calculate,

$$
\begin{gathered}
d\left(\left(\alpha_{i, i}\right)^{k}\left(\alpha_{j, j}\right)^{p-k-2}\left(\alpha_{i, j}\right)^{2}\right)=\left(\alpha_{i, i}\right)^{k}\left(\alpha_{j, j}\right)^{p-k-2}\left(2 \alpha_{i, j} d \alpha_{i, j}+d\left[\alpha_{i, j}\right]_{t}\right)= \\
\left(\alpha_{i, i}\right)^{k+1}\left(\alpha_{j, j}\right)^{p-k-1}\left|\xi_{i, j}\right|^{2} d t .
\end{gathered}
$$

We may clearly assume $\alpha_{1,1} \geq \alpha_{2,2} \geq \ldots \geq \alpha_{n, n}$, which implies that for $i<j$ and for all values of $k$, one has

$$
d\left(\left(\alpha_{i, i}\right)^{k}\left(\alpha_{j, j}\right)^{p-k-2}\left(\alpha_{i, j}\right)^{2}\right) \leq\left(\alpha_{i, i}\right)^{p}\left|\xi_{i, j}\right|^{2} d t
$$

Inspect the equation (32). For every $1 \leq i \leq n$, the expansion on the right hand side contains exactly one term of the first type, and for every distinct $i, j$ with $i \neq j$, it contains $\frac{p(p-1)}{2}$ terms of the second type (or otherwise, for all choices such that $i<j$, it contains $p(p-1)$ terms of this type). Using (33) and (34), we conclude

$$
\begin{gathered}
d S_{t} \leq \sum_{i=1}^{n} p \alpha_{i, i}^{p}\left\langle\xi_{i, i}, d W_{t}\right\rangle+p(p-1) \alpha_{i, i}^{p}\left|\xi_{i, i}\right|^{2} d t+\sum_{\substack{1 \leq i, j \leq n \\
i<j}} p(p-1)\left(\alpha_{i, i}\right)^{p}\left|\xi_{i, j}\right|^{2} d t \leq \\
\sum_{i=1}^{n} p \alpha_{i, i}^{p}\left\langle\xi_{i, i}, d W_{t}\right\rangle+p^{2} \sum_{i=1}^{n} \alpha_{i, i}^{p} \sum_{j=1}^{n}\left|\xi_{i, j}\right|^{2} d t \leq \\
\sum_{i=1}^{n} p \alpha_{i, i}^{p}\left\langle\xi_{i, i}, d W_{t}\right\rangle+p^{2} S_{t} K_{n}^{2} d t
\end{gathered}
$$

where in the last inequality we used the part (ii) of lemma 3.2

A well-known property of Itô processes is existence and uniqueness of the decomposition $S_{t}=M_{t}+E_{t}$, where $M_{t}$ is a local martingale and $E_{t}$ is an adapted process of locally bounded variation. In the last equation, we attained,

$$
d E_{t} \leq p^{2} K_{n}^{2} S_{t} d t
$$

and also,

$$
\frac{d[S]_{t}}{d t}=\left|\sum_{i=1}^{n} p \alpha_{i, i}^{p} \xi_{i, i}\right|^{2} .
$$

Using part (i) of lemma 3.2 yields,

$$
\frac{d[S]_{t}}{d t} \leq C p^{2} S_{t}^{2}
$$


Next, we use the unique decomposition $\log S_{t}=Y_{t}+Z_{t}$ where $Y_{t}$ is a local martingale, $Z_{t}$ is an adapted process of locally bounded variation and $Y_{0}=0$. According to Itô's formula and formula (36),

$$
\frac{d[Y]_{t}}{d t}=\frac{1}{S_{t}^{2}} \frac{d[S]_{t}}{d t} \leq C p^{2} .
$$

By Dambis / Dubins-Schwartz theorem, we know that there exists a standard Wiener process $\tilde{W}_{t}$ such that $Y_{t}$ has the same distribution as $\tilde{W}_{[Y]_{t}}$. An application of the so-called reflection principle gives,

$$
\begin{gathered}
\mathbb{P}\left(\max _{t \in[0, p]} \tilde{W}_{t} \geq t p\right)= \\
2 \mathbb{P}\left(\tilde{W}_{p} \geq t p\right)<C e^{-\frac{1}{2} t^{2} p} .
\end{gathered}
$$

Choosing $t$ to be a large enough universal constant, $C_{1}$, yields

$$
\mathbb{P}\left(\max _{t \in[0, p]} \tilde{W}_{t} \geq C_{1} p\right)<e^{-10 p},
$$

(where we used the fact that $p \geq 1$ ). Using (37), we attain

$$
\mathbb{P}\left(\max _{t \in\left[0, \frac{1}{p}\right]} Y_{t}>C_{2} p\right)<e^{-10 p}
$$

for some universal constant $C_{2}>0$. We now use Itô's formula again, this time with formula (35), to get

$$
\frac{d}{d t} Z_{t}=\frac{1}{S_{t}} \frac{d}{d t} E_{t}-\frac{1}{2 S_{t}^{2}} \frac{d[S]_{t}}{d t} \leq K_{n}^{2} p^{2}
$$

The last two equations and the legitimate assumption that $K_{n} \geq 1$ give,

$$
\mathbb{P}\left(\max _{t \in\left[0, \frac{1}{K_{n}^{2} p}\right]} \log S_{t}-\log n>C p\right)<e^{-10 p} .
$$

We choose $p=\lceil\log n\rceil$ to get,

$$
\mathbb{P}\left(\max _{t \in\left[0, \frac{1}{K_{n}^{2} \log n}\right]} S_{t}^{1 /\lceil\log n\rceil}>C^{\prime}\right)<\frac{1}{n^{10}},
$$

for some universal constant $C^{\prime}>0$. Define the event $F$ as the complement of the event in the equation above,

$$
F:=\left\{\max _{t \in\left[0, \frac{1}{K_{n}^{2} \log n}\right]} S_{t}^{1 /\lceil\log n\rceil} \leq C^{\prime}\right\} .
$$

Clearly, whenever the event $F$ holds, we have,

$$
\left\|A_{t}\right\|_{O P} \leq\left\|\tilde{A}_{t}\right\|_{O P} \leq C^{\prime}, \quad \forall t \in\left[0, \frac{1}{K_{n}^{2} \log n}\right]
$$


Our next task is to bound the norm for larger values of $t$. To this end, recall the bound (17). Recalling that $B_{t}=\int_{0}^{t} A_{s}^{-1} d s$, and applying (17) gives,

$$
\frac{d}{d t} B_{t}=A_{t}^{-1} \geq \frac{I d}{\Theta\left\|B_{t}^{-1}\right\|_{O P}}
$$

So,

$$
\frac{d}{d t} \frac{1}{\left\|B_{t}^{-1}\right\|_{O P}} \geq \frac{1}{\Theta\left\|B_{t}^{-1}\right\|_{O P}} .
$$

By the definition of $B_{t}$ and by (38), it follows that whenever $F$ holds one has,

$$
\frac{1}{\left\|B_{\delta^{2}}^{-1}\right\|_{O P}} \geq C \delta^{2}
$$

where $\delta^{2}=\frac{1}{K_{n}^{2} \log n}$. Equations (39) and (40) imply,

$$
B_{t} \geq c \delta^{2} e^{\left(t-\delta^{2}\right) / \Theta} I d, \quad \forall t>\delta^{2}
$$

which gives, using (17),

$$
A_{t} \leq C \delta^{-2} e^{\left(\delta^{2}-t\right) / \Theta} I d .
$$

Part(i) of the proposition is established. In order to prove the bound for $\mathbb{E}\left[\operatorname{Tr}\left(A_{t}\right)\right]$, write $S_{t}=\sum_{i=1}^{n} \operatorname{Tr}\left(\tilde{A}_{t}\right)$. Setting $p=1$ in (31) gives, $\frac{d}{d t} \mathbb{E}\left[S_{t}\right]=0$, which implies (ii). Part (iii) of the proposition follows directly from equations (40) and (12). The proposition is complete. 3.1

Proposition 2.6 gives an immediate corollary to part (iii) of proposition

Corollary 3.3 There exist universal constants $c, \Theta>0$ such that whenever the event $F$ defined in (20) holds, the following also holds:

Define $\delta=\frac{1}{K_{n} \sqrt{\log n}}$. Let $t>\delta^{2}$ and let $E \subset \mathbb{R}^{n}$ be a measurable set which satisfies,

$$
0.1 \leq \int_{E} f_{t}(x) d x \leq 0.9
$$

One has,

$$
\int_{E_{\Theta / \delta} \backslash E} f_{t}(x) d x \geq c
$$

where $E_{\Theta / \delta}$ is the $\frac{\Theta}{\delta}$-extension of $E$, defined in the introduction.

\section{Thin shell implies spectral gap}

In this section we use the localization scheme constructed in the previous sections in order to prove theorem 1.1 .

Let $f(x)$ be an isotropic log-concave probability density in $\mathbb{R}^{n}$ and let $E \subset \mathbb{R}^{n}$ be a measurable set. Suppose that,

$$
\int_{E} f(x) d x=\frac{1}{2}
$$


Our goal in this section is to show that,

$$
\int_{E_{\Theta / \delta} \backslash E} f(x) d x \geq c
$$

for some universal constants $c, \Theta>0$, where $\delta=\frac{1}{K_{n} \sqrt{\log n}}$ and $E_{\Theta / \delta}$ is the $\frac{\Theta}{\delta}$-extension of $E$.

The idea is quite simple. Define $f_{t}:=\Gamma_{t}(f)$, the localization of $f$ constructed in section 2, and fix $t>0$. By the martingale property of the localization, we have,

$$
\int_{E_{\Theta / \delta} \backslash E} f(x) d x=\mathbb{E}\left[\int_{E_{\Theta / \delta} \backslash E} f_{t}(x) d x\right] .
$$

Corollary 3.3 suggests that if $t$ is large enough, the right term can be bounded from below if we only manage to bound the integral $\int_{E} f_{t}(x) d x$ away from 0 and from 1.

Define,

$$
g(t)=\int_{E} f_{t}(x) d x
$$

In view of the above, we would like to prove:

Lemma 4.1 There exists a universal constant $T>0$ such that,

$$
\mathbb{P}(0.1 \leq g(t) \leq 0.9)>0.5, \quad \forall t \in[0, T] .
$$

Proof:

We calculate, using (13),

$$
d g(t)=\int_{E} f_{t}(x)\left\langle x-a_{t}, A_{t}^{-1 / 2} d W_{t}\right\rangle d x=
$$

(substitute $y=A_{t}^{-1 / 2}\left(x-a_{t}\right)$ )

$$
\begin{aligned}
& \sqrt{\operatorname{det} A_{t}} \int_{A_{t}^{-1 / 2}\left(E-a_{t}\right)} f_{t}\left(A_{t}^{1 / 2} y+a_{t}\right)\left\langle y, d W_{t}\right\rangle d y= \\
& \left\langle\sqrt{\operatorname{det} A_{t}} \int_{A_{t}^{-1 / 2}\left(E-a_{t}\right)} f_{t}\left(A_{t}^{1 / 2} y+a_{t}\right) y d y, d W_{t}\right\rangle .
\end{aligned}
$$

Define,

$$
\tilde{f}_{t}=\sqrt{\operatorname{det} A_{t}} f_{t}\left(A_{t}^{1 / 2} y+a_{t}\right), \quad E_{t}=A_{t}^{-1 / 2}\left(E-a_{t}\right)
$$

The above equation becomes,

$$
d g(t)=\left\langle\int_{E_{t}} y \tilde{f}_{t}(y) d y, d W_{t}\right\rangle .
$$


Assume, for now, that $\int_{E_{t}} y \tilde{f}_{t}(y) d y \neq 0$ and define $\theta=\frac{\int_{E_{t}} y \tilde{f}_{t}(y) d y}{\left|\int_{E_{t}} y \tilde{f}_{t}(y) d y\right|}$. Observe that, by definition, $\tilde{f}_{t}$ is isotropic. Consequently,

$$
\begin{gathered}
\left|\int_{E_{t}} y \tilde{f}_{t}(y) d y\right|=\left|\int_{E_{t}}\langle y, \theta\rangle \tilde{f}_{t}(y) d y\right| \leq \\
\int_{E_{t}}|\langle y, \theta\rangle| \tilde{f}_{t}(y) d y \leq \sqrt{\int_{E_{t}}\langle y, \theta\rangle^{2} \tilde{f}_{t}(y) d y} \leq 1 .
\end{gathered}
$$

We therefore learn that,

$$
\frac{d}{d t}[g]_{t} \leq 1, \quad \forall t>0
$$

Define $h(t)=(g(t)-0.5)^{2}$. By Itô's formula,

$$
d h(t)=2(g(t)-0.5) d g(t)+d[g]_{t} .
$$

Plugging the last two equations together gives,

$$
E\left[(g(t)-0.5)^{2}\right] \leq t .
$$

The lemma follows from an application of Chebyshev's inequality.

The last ingredient needed for our proof is a theorem of E. Milman, Mil2, Theorem 2.1]. The following is a weaker formulation of this theorem which will be suitable for us:

Theorem 4.2 (E. Milman) Suppose that a log-concave probability measure $\mu$ satisfies the following: there exist two constants, $0<\lambda<\frac{1}{2}$ and $\Theta>0$, such that for all measurable $E \subset \mathbb{R}^{n}$ with $\mu(E) \geq \frac{1}{2}$, one has $\mu\left(E_{\Theta}\right) \geq 1-\lambda$. In this case, the measure $\mu$ satisfies the following isoperimetric inequality:

For all measurable $E \subset \mathbb{R}^{n}$ with $\mu(E) \leq \frac{1}{2}$,

$$
\frac{\mu^{+}(E)}{\mu(E)} \geq \frac{1-2 \lambda}{\Theta}
$$

Note that equation (48) is the exact type of inequality defining the constant $G_{n}$ in equation (2). We are now ready to prove the main proposition of this section.

Proof of proposition 1.7

Let $T$ be the constant from lemma 4.1. Denote,

$$
G=\{0.1 \leq g(T) \leq 0.9\} \cap F .
$$

where $F$ is the event defined in (20). According to lemma 4.1 and to (21), one has $\mathbb{P}(G)>0.4$ for all $n \geq 2$. 
By (45) and by corollary 3.3. there exist universal constants $\tilde{c}, \Theta>0$ such that

$$
\begin{gathered}
\int_{E_{\Theta / \delta} \backslash E} f(x) d x=\mathbb{E}\left[\int_{E_{\Theta / \delta} \backslash E} f_{T}(x) d x\right] \geq \\
P(G) \mathbb{E}\left[\int_{E_{\Theta / \delta} \backslash E} f_{T}(x) d x \mid G\right] \geq \tilde{c} .
\end{gathered}
$$

The result now follows directly from an application of theorem 4.2 .

Remark 4.3 In the above proof, we used E. Milman's result in order to reduce the theorem to the case where $\int_{E} f(x) d x$ is exactly $\frac{1}{2}$, as well as to attain an isoperimetric inequality from a certain concentration inequality for distance functions. Alternatively, we may have replaced propsition 2.6 with an essentially stronger result due to Bakry-Emery, proven in [BE] (see also Gross, Gros1]). Their result, which relies on the hypercontractivity principle, asserts that a density of the form (12) actually possesses a respective Cheeger constant. Using this fact, we may have directly bounded from below the surface area of any set with respect to the measure whose density is $f_{t}$.

The proof of lemma 1.6 is in section 6 . Along with this lemma, we have established theorem 1.1

\section{$5 \quad$ Stability of the Brunn-Minkowski in- equality}

The main goal of this section is to prove theorem 1.2

The idea of the proof is as follows: Given two log-concave densities, $f$ and $g$, we run the localization process we constructed in section 2 on both functions, so that their corresponding localization processes are coupled together in the sense that we take the same Wiener process $W_{t}$ for both functions. Recall formula (19), whose point is that the barycenters of the localized functions $f_{t}$ and $g_{t}$ converge, in the Wasserstein metric, to the measures whose densities are $f$ and $g$, respectively. In view of this, it is enough to consider the paths of the barycenters and show that they remain close to each other along the process. Recall that if $a_{t}$ is the barycenter of $f_{t}$, we have $d a_{t}=A_{t}^{1 / 2} d W_{t}$. This formula tells us that as long as we manage to keep the covariance matrices of $f_{t}$ and $g_{t}$ approximately similar to each other, the barycenters will not move too far apart. In order to do this, we use an idea from [EK2]: when the integral of the supremum convolution of two given densities is rather small, these densities can essentially be regarded as parallel sections of an isotropic convex body, which means, by thin-shell concentration, that the corresponding covariance matrices cannot be very different from each other. 
We begin with some notation. For two functions $f, g: \mathbb{R}^{n} \rightarrow \mathbb{R}^{+}$, denote by $H_{\lambda}(f, g)$ the supremum convolution of the two functions, hence,

$$
H(f, g)(x):=\sup _{y \in \mathbb{R}^{n}} \sqrt{f(x+y) g(x-y)} .
$$

Next, define,

$$
K(f, g)=\int_{\mathbb{R}^{n}} H(f, g)(x) d x .
$$

The following lemma is a variant of lemma 6.5 from EK2].

Lemma 5.1 There exists a universal constant $C>0$ such that the following holds: Let $f, g$ be log-concave probability densities in $\mathbb{R}^{n}$. Define,

$$
A=\operatorname{Cov}(f)^{-1 / 2} \operatorname{Cov}(g) \operatorname{Cov}(f)^{-1 / 2}-I d,
$$

and let $\left\{\delta_{i}\right\}_{i=1}^{n}$ be the eigenvalues of $A$ such that the order of $\left|\delta_{i}-1\right|$ is decreasing. Then,

$$
\left|\delta_{i}-1\right| \leq C K(f, g)^{4}, \quad \forall 1 \leq i \leq n
$$

and,

$$
\left|\delta_{i}-1\right| \leq C K(f, g) \tau_{n} i^{\kappa-\frac{1}{2}}, \quad \forall(\log K(f, g))^{C_{1}} \leq i \leq n
$$

where $C, C_{1}>0$ are universal constants.

Our main ideas in this section are contained in the following lemma:

Lemma 5.2 Let $\epsilon>0$ and let $f, g$ be log-concave probability densities in $\mathbb{R}^{n}$ such that $f$ is isotropic and the barycenter of $g$ lies at the origin. In that case, there exist two densities, $\tilde{f}, \tilde{g}$, which satisfy,

and,

$$
\begin{gathered}
\tilde{f}(x) \leq f(x), \quad \tilde{g}(x) \leq g(x), \quad \forall x \in \mathbb{R}^{n}, \\
\int_{\mathbb{R}^{n}} \tilde{f}(x) d x=\int_{\mathbb{R}^{n}} \tilde{g}(x) d x \geq 1-\epsilon
\end{gathered}
$$

$$
W_{2}(\tilde{f}, \tilde{g}) \leq \frac{C}{\epsilon^{6}} \tau_{n} K(f, g)^{5} n^{2\left(\kappa-\kappa^{2}\right)+\epsilon}
$$

Proof: As explained in the beginning of the section, we will couple between the measures $f$ and $g$ in means of coupling between the processes $\Gamma_{t}(f)$ and $\Gamma_{t}(g)$. To that end, we define, as in (13),

$$
F_{0}(x)=1, \quad d F_{t}(x)=\left\langle A_{t}^{-1 / 2} d W_{t}, x-a_{t}\right\rangle F_{t}(x)
$$

where,

$$
a_{t}=\frac{\int_{\mathbb{R}^{n}} x f(x) F_{t}(x) d x}{\int_{\mathbb{R}^{n}} f(x) F_{t}(x) d x}
$$

is the barycenter of $f F_{t}$, and,

$$
A_{t}=\int_{\mathbb{R}^{n}}\left(x-a_{t}\right) \otimes\left(x-a_{t}\right) f(x) F_{t}(x) d x
$$


is the covariance matrix of $f F_{t}$. As usual denote $f_{t}=F_{t} f$.

Next, we define,

$$
G_{0}(x)=1, \quad d G_{t}(x)=\left\langle A_{t}^{-1 / 2} d W_{t}, x-b_{t}\right\rangle G_{t}(x)
$$

where,

$$
b_{t}=\frac{\int_{\mathbb{R}^{n}} x g(x) G_{t}(x) d x}{\int_{\mathbb{R}^{n}} g(x) G_{t}(x) d x},
$$

and denote $g_{t}(x)=g(x) G_{t}(x)$.

Finally, we "interpolate" between the two processes by defining,

$$
H_{0}(x)=1, \quad d H_{t}(x)=\left\langle A_{t}^{-1 / 2} d W_{t}, x-\left(a_{t}+b_{t}\right) / 2\right\rangle,
$$

and,

$$
h_{t}(x)=H_{t}(x) H(f, g)(x) .
$$

By a similar calculation to the one carried out in lemma 2.4, we learn that for all $t \geq 0, \int f_{t}(x) d x=\int g_{t}(x) d x=1$. Fix $x, y \in \mathbb{R}^{n}$. An application of Itô's formula yields

$$
\begin{aligned}
& d \log f_{t}(x+y)=\left\langle x+y-a_{t}, A_{t}^{-1 / 2} d W_{t}\right\rangle-\frac{1}{2}\left|A_{t}^{-1 / 2}\left(x+y-a_{t}\right)\right|^{2} d t, \\
& d \log g_{t}(x-y)=\left\langle x-y-b_{t}, A_{t}^{-1 / 2} d W_{t}\right\rangle-\frac{1}{2}\left|A_{t}^{-1 / 2}\left(x-y-b_{t}\right)\right|^{2} d t
\end{aligned}
$$

and

$$
d \log h_{t}(x)=\left\langle x-\frac{a_{t}+b_{t}}{2}, A_{t}^{-1 / 2} d W_{t}\right\rangle-\frac{1}{2}\left|A_{t}^{-1 / 2}\left(x-\left(a_{t}+b_{t}\right) / 2\right)\right|^{2} d t .
$$

Consequently,

$$
2 d \log h_{t}(x) \geq d \log f_{t}(x+y)+d \log g_{t}(x-y) .
$$

It follows that,

$$
h_{t}(x) \geq H\left(f_{t}, g_{t}\right)(x) .
$$

Define $S_{t}=\int_{\mathbb{R}^{n}} h_{t}(x) d x$. The definition of $H_{t}$ suggests that $S_{t}$ is a martingale. By the Dambis / Dubins-Schwarz theorem, there exists a nondecreasing function $A(t)$ such that,

$$
S_{t}=K(f, g)+\tilde{W}_{A(t)}
$$

where $\tilde{W}_{t}$ is distributed as a standard Wiener process. Since $S_{t} \geq 1$ almost surely, it follows from the Doob's maximal inequality theorem that,

$$
\mathbb{P}\left(G_{t}\right) \geq 1-\epsilon / 2, \quad \forall s>0 .
$$

where,

$$
G_{t}=\left\{\max _{s \in[0, t]} S_{s} \leq \frac{2 K(f, g)}{\epsilon}\right\} .
$$

Next, define,

$$
F_{t}:=\left\{\left\|A_{s}\right\|_{O P}<C K_{n}^{2}(\log n) e^{-t}, \quad \forall 0 \leq s \leq t\right\} .
$$


where $C$ is the same constant as in (20). Finally, denote $E_{t}=G_{t} \cap F_{t}$. By proposition 3.1 and equation (54), $P\left(E_{t}\right)>1-\epsilon$ for all $t>0$. Define a stopping time by the equation,

$$
\rho=\sup \left\{t \mid E_{t} \text { holds }\right\} .
$$

Our next objective is to define the densities $\tilde{f}, \tilde{g}$ by, in some sense, neglecting the cases where $E_{t}$ does not hold. We begin by defining the density $\tilde{f}_{t}$ by the following equation,

$$
\int_{B} \tilde{f}_{t}(x) d x=\mathbb{E}\left[\mathbf{1}_{E_{t}} \int_{B} f_{t}(x) d x\right],
$$

for all measurable $B \subset \mathbb{R}^{n}$. Likewise, we define

$$
\int_{B} \tilde{g}_{t}(x) d x=\mathbb{E}\left[\mathbf{1}_{E_{t}} \int_{B} g_{t}(x) d x\right] .
$$

Recall that $f(x)=\mathbb{E}\left[f_{t}(x)\right]$ for all $x \in \mathbb{R}^{n}$ and $t>0$. It follows that,

$$
\int_{\mathbb{R}^{n}} \tilde{f}_{t}(x) d x=\int_{\mathbb{R}^{n}} \tilde{g}_{t}(x) d x=P\left(E_{t}\right) \geq 1-\epsilon,
$$

and that

$$
\tilde{f}_{t}(x) \leq f(x), \quad \tilde{g}_{t}(x) \leq g(x), \quad \forall x \in \mathbb{R}^{n}
$$

We construct a coupling between $\tilde{f}_{t}$ and $\tilde{g}_{t}$ by defining a measure $\mu_{t}$ on $\mathbb{R}^{n} \times \mathbb{R}^{n}$ using the formula

$$
\mu_{t}(A \times B)=\mathbb{E}\left[\mathbf{1}_{E_{t}} \int_{A \times B} f_{t}(x) g_{t}(y) d x d y\right],
$$

for any measurable sets $A, B \subset \mathbb{R}^{n}$. It is easy to check that $\tilde{f}_{t}$ and $\tilde{g}_{t}$ are the densities of the marginals of $\mu_{t}$ onto its first and last $n$ coordinates respectively. Thus, by definition of the Wasserstein distance,

$$
\begin{gathered}
W_{2}\left(\tilde{f}_{t}, \tilde{g}_{t}\right) \leq\left(\int_{\mathbb{R}^{n} \times \mathbb{R}^{n}}|x-y|^{2} d \mu_{t}(x, y)\right)^{1 / 2}= \\
\left(\mathbb{E}\left[\mathbf{1}_{E_{t}} \int_{\mathbb{R}^{n} \times \mathbb{R}^{n}}|x-y|^{2} f_{t}(x) g_{t}(y) d x d y\right]\right)^{1 / 2} \leq \\
\left(\mathbb{E}\left[\mathbf{1}_{E_{t}}\left(W_{2}\left(f_{t}, a_{t}\right)+W_{2}\left(g_{t}, b_{t}\right)+\left|a_{t}-b_{t}\right|\right)^{2}\right]\right)^{1 / 2} .
\end{gathered}
$$

Now, thanks to formula (19), we can take $T$ large enough (and deterministic) such that,

$$
\begin{gathered}
W_{2}\left(\tilde{f}_{T}, \tilde{g}_{T}\right) \leq 2\left(\mathbb{E}\left[\mathbf{1}_{E_{T}}\left|a_{T}-b_{T}\right|^{2}\right]\right)^{1 / 2}+1 \leq \\
2\left(\mathbb{E}\left[\left|a_{T \wedge \rho}-b_{T \wedge \rho}\right|^{2}\right]\right)^{1 / 2}+1 .
\end{gathered}
$$


We will define $\tilde{f}:=\tilde{f}_{T}$ and $\tilde{g}:=\tilde{g}_{T}$. In view of the last equation, our main goal will be to attain a bound for the process $\left|a_{t}-b_{t}\right|$. A similar calculation to the one carried out in (16) gives,

$$
d a_{t}=A_{t}^{1 / 2} d W_{t}, \quad d b_{t}=C_{t} A_{t}^{-1 / 2} d W_{t} .
$$

where,

$$
C_{t}=\int_{\mathbb{R}^{n}}\left(x-b_{t}\right) \otimes\left(x-b_{t}\right) g_{t}(x) d x
$$

is the covariance matrix of $g_{t}$. Therefore,

$$
\begin{gathered}
d\left|a_{t}-b_{t}\right|^{2}=2\left\langle a_{t}-b_{t}, d a_{t}\right\rangle-2\left\langle a_{t}-b_{t}, d b_{t}\right\rangle+ \\
\left\langle d a_{t}, d a_{t}\right\rangle+\left\langle d b_{t}, d b_{t}\right\rangle-2\left\langle d a_{t}, d b_{t}\right\rangle .
\end{gathered}
$$

The first two terms are martingale. We use the unique decomposition

$$
\left|a_{t}-b_{t}\right|^{2}=M_{t}+N_{t}
$$

where $M_{t}$ is a local martingale and $N_{t}$ is an adapted process of locally bounded variation. We get,

$$
\begin{gathered}
\frac{d}{d t} N_{t}=\left\langle d a_{t}-d b_{t}, d a_{t}-d b_{t}\right\rangle= \\
\left\langle\left(A_{t}-C_{t}\right) A_{t}^{-1 / 2} d W_{t},\left(A_{t}-C_{t}\right) A_{t}^{-1 / 2} d W_{t}\right\rangle= \\
\left\|A_{t}^{1 / 2}\left(I-A_{t}^{-1 / 2} C_{t} A_{t}^{-1 / 2}\right)\right\|_{H S}^{2} .
\end{gathered}
$$

By the Optional Stopping Theorem,

$$
\mathbb{E}\left[\left|a_{t \wedge \rho}-b_{t \wedge \rho}\right|^{2}\right]=\mathbb{E}\left[N_{t \wedge \rho}\right]=\mathbb{E}\left[\int_{0}^{t \wedge \rho}\left\|D_{s}\right\|_{H S}^{2} d s\right]
$$

where $D_{t}=A_{t}^{1 / 2}\left(I-A_{t}^{-1 / 2} C_{t} A_{t}^{-1 / 2}\right)$. Our next task is to use lemma 5.1 to bound $\left\|D_{t}\right\|_{H S}$ under the assumption $\mathrm{f} t<\tau$.

We start by denoting the eigenvalues of the matrix $I-A_{t}^{-1 / 2} C_{t} A_{t}^{-1 / 2}$ by $\delta_{i}$, in decreasing order, and the eigenvalues of the matrix $A_{t}$ by $\lambda_{i}$, also in decreasing order. By theorem 1 in $\mathrm{T}$,

$$
\left\|D_{t}\right\|_{H S}^{2} \leq \sum_{j=1}^{n} \lambda_{j} \delta_{j}^{2}
$$

By lemma 5.1, we learn that

$$
\delta_{j} \leq \frac{C K\left(f_{t}, g_{t}\right)^{5} \tau_{n} j^{\kappa}}{\sqrt{j}}
$$

Plugging this into (59) yields,

$$
\left\|D_{t}\right\|_{H S}^{2} \leq C K\left(f_{t}, g_{t}\right)^{10} \tau_{n}^{2} \sum_{j=1}^{n} \lambda_{j} j^{2 \kappa-1}
$$


Fix some constant $(1-2 \kappa)<\alpha<1$, whose value will be chosen later. For now, we assume that $\kappa>0$. Using Hölder's inequality, we calculate,

$$
\begin{gathered}
\left\|D_{t}\right\|_{H S}^{2} \leq C K\left(f_{t}, g_{t}\right)^{10} \tau_{n}^{2}\left(\sum_{j=1}^{n} \lambda_{j}^{1 /(1-\alpha)}\right)^{1-\alpha}\left(\sum_{j=1}^{n} j^{(2 \kappa-1) / \alpha}\right)^{\alpha} \leq \\
C K\left(f_{t}, g_{t}\right)^{10} \tau_{n}^{2}\left(\lambda_{1}^{1 /(1-\alpha)-1} \sum_{j=1}^{n} \lambda_{j}\right)^{1-\alpha}\left(1+\int_{1}^{n} t^{(2 \kappa-1) / \alpha}\right)^{\alpha} \leq \\
C K\left(f_{t}, g_{t}\right)^{10} \tau_{n}^{2} \lambda_{1}^{\alpha}(\beta n)^{1-\alpha}\left(n^{(2 \kappa-1) / \alpha+1}+2\right)^{\alpha}\left(\frac{1}{(2 \kappa-1) / \alpha+1}\right)^{\alpha}
\end{gathered}
$$

where $\beta=\frac{1}{n} \sum_{j=1}^{n} \lambda_{j}$. Recall that $\alpha>(1-2 \kappa)$, which gives,

$$
\left(n^{(2 \kappa-1) / \alpha+1}+2\right)^{\alpha} \leq 3 n^{\alpha} n^{2 \kappa-1} .
$$

Take $\alpha$ such that $\epsilon=\alpha-(1-2 \kappa)$. Equations (61) and (62) give,

$$
\begin{gathered}
\left\|D_{t}\right\|_{H S}^{2} \leq \frac{C^{\prime}}{\epsilon} K\left(f_{t}, g_{t}\right)^{10} \tau_{n}^{2} \beta^{1-\alpha} \lambda_{1}^{\alpha} n^{2 \kappa} \leq \\
\frac{C^{\prime \prime}}{\epsilon} K\left(f_{t}, g_{t}\right)^{10} \tau_{n}^{2} \max (\beta, 1) \lambda_{1}^{1-2 \kappa+\epsilon} n^{2 \kappa} .
\end{gathered}
$$

Recall that we assume that $t<\tau$. By the definition of $\tau$, we get $\lambda_{1} \leq$ $C \tau_{n}^{2} n^{2 \kappa} \log n$ and $K\left(f_{t}, g_{t}\right) \leq 2 K(f, g) / \epsilon$. Part (ii) of proposition 3.1 implies $\mathbb{E}[\beta] \leq 1$. Plugging these facts into the last equation gives,

$$
\begin{aligned}
\mathbb{E}\left[\left\|D_{t}\right\|_{H S}^{2}\right] \leq & \frac{C}{\epsilon^{11}} K(f, g)^{10} \tau_{n}^{2}\left(\tau_{n}^{2} n^{2 \kappa} \log n\right)^{1-2 \kappa+\epsilon} n^{2 \kappa} e^{-t} \leq \\
& \leq \frac{C^{\prime}}{\epsilon^{11}} K(f, g)^{10} \tau_{n}^{2} n^{4 \kappa-4 \kappa^{2}+\epsilon} e^{-t} .
\end{aligned}
$$

Finally, using equations (56) and (58), we conclude,

$$
\begin{gathered}
W_{2}\left(\tilde{f}_{T}, \tilde{g}_{T}\right)^{2} \leq \mathbb{E}\left[\int_{0}^{T \wedge \rho}\left\|D_{s}\right\|_{H S}^{2} d s\right] \leq \\
\frac{C}{\epsilon^{11}} K(f, g)^{10} \tau_{n}^{2} n^{4 \kappa-4 \kappa^{2}+\epsilon} .
\end{gathered}
$$

The proof is complete.

Remark 5.3 In the above lemma, if we replace the assumption that $f$ is isotropic by the assumption that $f, g$ are log-concave with respect to the Gaussian measure, then following the same lines of proof while using proposition 2.6. one may improve the bound (52) and get,

$$
W_{2}(\tilde{f}, \tilde{g}) \leq C(\epsilon) K(f, g) \sqrt{\log n} .
$$


We move on to the proof of theorem 1.2

Proof of theorem 1.2: Let $K, T$ be convex bodies of volume 1 such that the covariance matrix of $K$ is $L_{k}^{2} I d$. Fix $\epsilon>0$. Define,

$$
f(x)=1_{K / L_{K}}(x) L_{K}^{n}, \quad g(x)=1_{T / L_{K}}(x) L_{K}^{n},
$$

so both $f$ and $g$ are probability measures and $f$ is isotropic. We have,

$$
K(f, g)=\operatorname{Vol}_{n}\left(\frac{K+T}{2}\right)=V .
$$

We use lemma 5.2, which asserts that there exist two measures $\tilde{f}, \tilde{g}$, such that,

$$
\begin{gathered}
\tilde{f}(x) \leq f(x), \quad \tilde{g}(x) \leq g(x), \quad \forall x \in \mathbb{R}^{n}, \\
\int \tilde{f}(x) d x=\int \tilde{g}(x) d x \geq 1-\epsilon
\end{gathered}
$$

and such that,

$$
W_{2}(\tilde{f}, \tilde{g}) \leq \Theta
$$

where $\Theta=C(\epsilon) V^{5} \tau_{n} n^{2\left(\kappa-\kappa^{2}\right)+\epsilon}$. Since $\tilde{g}$ is supported on $T$, it follows that,

where $d\left(x, T / L_{T}\right)=\inf _{y \in\left(T / L_{T}\right)}|x-y|$. Denote,

$$
\int_{K} d^{2}\left(x, T / L_{T}\right) \tilde{f}(x) d x \leq \Theta^{2}
$$

$$
K_{\alpha}=\left\{x \in K / L_{K} ; d(x, T) \geq \alpha \Theta\right\} .
$$

It follows from Markov's inequality and from (64) and 65 that,

$$
\operatorname{Vol}_{n}\left(K_{\alpha}\right) \leq L_{K}^{-n}\left(\epsilon+\frac{1}{\alpha^{2}}\right)
$$

Finally, taking $\delta=L_{K} \Theta / \sqrt{\epsilon}$ gives

$$
\operatorname{Vol}_{n}\left(K \backslash T_{\delta}\right) \leq 2 \epsilon .
$$

This completes the proof.

\section{$6 \quad$ Tying up loose ends}

We begin the section with the proof of lemma 1.6 which gives an upper bound for the constant $K_{n}$ in terms of $\tau_{n}$ and $\kappa$.

Proof of lemma 1.6; Let $X$ be an isotropic, log concave random vector in $\mathbb{R}^{n}$, and fix $\theta \in S^{n-1}$. Denote $A=\mathbb{E}[X \otimes X\langle X, \theta\rangle]$. Our goal is to show,

$$
\|A\|_{H S}^{2} \leq C \sum_{k=1}^{n} \frac{\sigma_{k}^{2}}{k} .
$$


Let $k \leq n$ and let $E_{k}$ be a subspace of dimension $k$. Denote $P(X)=$ $\operatorname{Proj}_{E_{k}}(X)$ and $Y=|P(X)|-\sqrt{k}$. By definition of $\sigma_{k}$,

$$
\operatorname{Var}[Y] \leq \sigma_{k}^{2}
$$

Note that, by the isotropicity of $X, \mathbb{E}\left[|P(X)|^{2}\right]=k$. It easily follows that,

$$
\operatorname{Var}\left[|P(X)|^{2}\right] \leq C k \operatorname{Var}[Y] \leq C k \sigma_{k}^{2} .
$$

Using the last inequality and applying Cauchy-Schwartz gives,

$$
\left|\mathbb{E}\left[\langle X, \theta\rangle|P(X)|^{2}\right]\right| \leq \sqrt{\operatorname{Var}[\langle X, \theta\rangle] \operatorname{Var}\left[|P(X)|^{2}\right]} \leq C \sqrt{k} \sigma_{k}
$$

or, in other words,

$$
\left|\operatorname{Tr}\left[\operatorname{Proj}_{E_{k}} A \operatorname{Proj}_{E_{k}}\right]\right| \leq C \sqrt{k} \sigma_{k} .
$$

Let $\lambda_{1}, \ldots, \lambda_{\ell}$ be the non-negative eigenvalues of $A$ in decreasing order. The last inequality implies that the matrix $\operatorname{Proj}_{E_{k}} \operatorname{AProj}_{E_{k}}$ has at least one eigenvalue smaller than $C \sqrt{\frac{1}{k}} \sigma_{k}$. Consequently, by taking $E_{k}$ to be the subspace spanned by the $k$ first corresponding eigenvectors we learn that

$$
\lambda_{k}^{2}<C \frac{\sigma_{k}^{2}}{k}, \quad \forall k \leq \ell
$$

In the same manner, if $\zeta_{1}, \ldots, \zeta_{n-\ell}$ are the negative eigenvalues of $A$, one has $\zeta_{k}^{2}<C \frac{\sigma_{k}^{2}}{k}$.

We can thus calculate,

$$
\|A\|_{H S}^{2}=\sum_{k=1}^{\ell} \lambda_{k}^{2}+\sum_{k=1}^{n-\ell} \zeta_{k}^{2} \leq 2 C \sum_{k=1}^{n} \frac{\sigma_{k}^{2}}{k}
$$

The proof is complete.

Next, in order to provide the reader with a better understanding of the constant $K_{n}$, we introduce two new constants. First, define

$$
Q_{n}^{2}=\sup _{X, Q} \frac{\operatorname{Var}[Q(X)]}{\mathbb{E}\left[|\nabla Q(X)|^{2}\right]}
$$

where the supremum runs over all isotropic log-concave random vectors, $X$, and all quadratic forms $Q(x)$. Next, define

$$
R_{n}^{-1}=\inf _{\mu, E} \frac{\mu^{+}(E)}{\mu(E)}
$$

where $\mu$ runs over all isotropic log-concave measures and $E$ runs over all ellipsoids with $\mu(E) \leq 1 / 2$.

Fact 6.1 There exist universal constants $C_{1}, C_{2}$ such that

$$
K_{n} \leq C_{1} Q_{n} \leq C_{2} R_{n}
$$


The proof of the right inequality is standard and uses the coarea formula and the Cauchy-Schwartz inequality. We will prove the left inequality. To that end, fix an isotropic log-concave random vector $X$, denote $A=$ $\mathbb{E}\left[X \otimes X X_{1}\right]$. We have,

$$
\|A\|_{H S}=\sup _{B} \frac{\operatorname{Tr}(B A)}{\|B\|_{H S}}
$$

where $B$ runs over all symmetric matrices. Let $B$ be a symmetric matrix. Fix coordinates under which $B$ is diagonal, and write $X=\left(X_{1}, \ldots, X_{n}\right)$ and $B=\operatorname{diag}\left\{a_{1}, . ., a_{n}\right\}$. Define $Q(x)=\langle B x, x\rangle$. We have,

$$
\begin{gathered}
\operatorname{Tr}(B A)=\mathbb{E}\left[X_{1} \sum_{i=1}^{n} a_{i} X_{i}^{2}\right] \leq \sqrt{\mathbb{E}\left[X_{1}^{2}\right]} \sqrt{\operatorname{Var}\left[\sum_{i=1}^{n} a_{i} X_{i}^{2}\right]}= \\
\sqrt{\operatorname{Var}[Q(X)]} \leq \sqrt{2 Q_{n}^{2} \sum_{i=1}^{n} a_{i}^{2} \mathbb{E}\left[X_{i}^{2}\right]}=\sqrt{2} Q_{n}\|B\|_{H S} .
\end{gathered}
$$

So,

$$
\|A\|_{H S} \leq \sqrt{2} Q_{n}
$$

This shows that $K_{n} \leq C Q_{n}$.

Remark 6.2 We suspect that there exists a universal constant $C>0$ such that $K_{n} \leq C \sigma_{n}$, but we are unable to prove that assertion.

We move on to the proof of lemma 2.5

Proof of lemma 2.5.

Throughout the proof, all the constants $c, c_{1}, c_{2}, \ldots$ may depend only on the dimension $n$. Recall that $f(x)$ is assumed to be isotropic and logconcave. It is well-known that there exist two constants $c_{1}, c_{2}>0$, such that

$$
f(|x|) \geq c_{1}, \quad \forall|x| \leq c_{2} .
$$

(see for example [LV, Theorem 5.14]). Define $g(x)=c_{1} \mathbf{1}_{\left\{|x| \leq c_{2}\right\}}$. It is also well-known (see for example [LV] Lemma 5.7]) that there exist two constants $c_{3}, c_{4}>0$ such that

$$
\int_{\mathbb{R}^{n}} f(x) e^{\langle x, y\rangle} d x \leq c_{3}, \quad \forall|y|<c_{4},
$$

which implies that whenever $|c|<c_{4}$ and $B$ is positive semi-definite,

$$
V_{f}(c, B)=\int_{\mathbb{R}^{n}} e^{\langle c, x\rangle-\frac{1}{2}\langle B x, x\rangle} f(x) d x \leq c_{3} .
$$

It follows that for all $|c|<c_{4}$ and $B \leq I d$ (in the sense of positive matrices), one has

$$
\begin{gathered}
A_{f}(c, B) \geq \\
c_{3}^{-1} \int_{\mathbb{R}^{n}}\left(x-a_{f}(c, B)\right) \otimes\left(x-a_{f}(c, B)\right) e^{-c_{4}|x|-\frac{1}{2}|x|^{2}} g(x) d x \geq \\
c_{3}^{-1} c_{1} \int_{\left\{|x| \leq c_{2}\right\}} x \otimes x e^{-c_{4}|x|-\frac{1}{2}|x|^{2}} d x=c_{5} I d
\end{gathered}
$$


for some constant $c_{5}>0$. Define the stopping times,

$T_{1}=\sup \left\{t>0 ;\left|c_{t}\right|<c_{4}\right\}, T_{2}=\sup \left\{t>0 ; B_{t} \geq I d\right\}, T=\min \left(T_{1}, T_{2}\right)$.

Note that according to (67),

$$
A_{t} \geq c_{5} I d, \quad \forall t \leq T
$$

so the lemma would be concluded if we manage to show that

$$
\mathbb{P}(T>c)>c
$$

for some constant $c>0$.

Define the event $E=\left\{T_{2} \leq T_{1}\right\}$. Whenever $E$ holds, we have the following: First, using (67),

$$
A_{t} \geq c_{5} I d, \quad \forall t \leq T_{2} .
$$

Recall that $\frac{d}{d t} B_{t}=A_{t}^{-1}$. It follows that

$$
B_{t} \leq c_{5}^{-1} t, \quad \forall t \leq T_{2}
$$

By taking $t=T_{2}$ in the last equation, we learn that $T=T_{2} \geq c_{5}$ whenever $E$ holds, so

$$
T_{2} \leq T_{1} \Rightarrow T \geq c_{5}
$$

Therefore, it is enough to prove that $P\left(T_{1}>c\right)>c$ for some $c>0$. Furthermore, in the following we are able to assume that $P(E) \leq 0.1$.

To that end, consider the defining equation (11) and use Itô's formula to attain

$$
d\left|c_{t}\right|^{2}=2\left\langle c_{t}, A_{t}^{-1 / 2} d W_{t}\right\rangle+2\left\langle A_{t}^{-1} a_{t}, c_{t}\right\rangle d t+\left\|A_{t}^{-1 / 2}\right\|_{H S}^{2} d t .
$$

Define the process $e_{t}$ by the equations,

$$
e_{t}=0, \quad d e_{t}=2\left\langle c_{t}, A_{t}^{-1 / 2} d W_{t}\right\rangle .
$$

Using (68), we deduce that whenever $t<T$, one has

$$
[e]_{t}=4 \int_{0}^{t}\left\langle A_{t}^{-1 / 2} c_{t}, A_{t}^{-1 / 2} c_{t}\right\rangle \leq 4 c_{4}^{2} c_{5}^{-1} t
$$

Using the Dambis / Dubins-Schwartz theorem, we know that there exists a standard Wiener process $\tilde{W}_{t}$ such that $e_{t}$ has the same distribution as $\tilde{W}_{[e]_{t}}$. An elementary property of the standard Wiener process is that there exists a constant $c_{6}>0$, such that

$$
\mathbb{P}(F) \geq 0.9
$$

where

$$
F=\left\{\max _{0 \leq s \leq c_{6}} \tilde{W}_{s} \leq c_{4}^{2} / 2\right\}
$$


Define $\delta=\min \left(T, \frac{c_{6}}{4 c_{4}^{2} c_{5}^{-1}}\right)$. Note that, by (71),

$$
F \subseteq\left\{\max _{0 \leq t \leq \delta} e_{t} \leq c_{4}^{2} / 2\right\} .
$$

Another application of (68), this time with the assumption (10) gives,

$$
\int_{0}^{t}\left\|A_{s}^{-1 / 2}\right\|_{H S}^{2} d s+2\left|\left\langle A_{s}^{-1} a_{s}, c_{s}\right\rangle\right| d s \leq n c_{5}^{-1}\left(1+c_{4}\right) t \leq c_{7} t
$$

for all $t<T$, and for a constant $c_{7}$. By plugging (73) and (74) into (70), we learn that whenever $F$ holds, one has

$$
\left|c_{t}\right|^{2} \leq c_{4}^{2} / 2+c_{7} t, \quad \forall t \leq \delta
$$

If we assume that $\delta=T_{1}$, the above gives $c_{4}^{2}=\left|c_{T_{1}}\right|^{2} \leq c_{4}^{2} / 2+c_{7} T_{1}$ which implies $T \geq \frac{c_{4}^{2}}{2 c_{7}}$ (here, we used the assumption that $T_{1} \leq T_{2}$ ). Thus, whenever $F \cap E^{C}$ holds, we have $T=T_{1} \geq \min \left(\frac{c_{4}^{2}}{2 c_{7}}, \frac{c_{6}}{4 c_{4}^{2} c_{5}^{-1}}\right)$. A union bound gives $\mathbb{P}\left(F \cap E^{C}\right) \geq 0.8$. The lemma is complete.

\section{References}

[ABP] M. Anttila, K. Ball, I. Perissinaki, The central limit problem for convex bodies. Trans. Amer. Math. Soc., 355, no. 12, (2003), 47234735 .

[BE] D. Bakry and M. Emery, Diffusions hypercontractives, in Séminaire de probabilités, XIX, 1983/84, vol. 1123 of Lecture Notes in Math., Springer, Berlin, 1985, pp. 177206.

[BN] K. Ball V.H. Ngyuen, Entropy jumps for random vectors with log-concave density and spectral gap. Preprint.

[Bo] S. Bobkov, On isoperimetric constants for log-concave probability distributions, in Geometric Aspects of Functional Analysis Israel Seminar 2004-2005, Springer Lecture Notes in Math. 1910 (2007), 8188.

[BK] S. Bobkov, A. Koldobsky, On the central limit property of convex bodies. Geometric aspects of functional analysis, Lecture Notes in Math., 1807, Springer, Berlin, (2003), 44-52.

[Bou] Bourgain, J., On the distribution of polynomials on highdimensional convex sets. Geometric aspects of functional analysis, Israel seminar (1989-90), Lecture Notes in Math., 1469, Springer, Berlin, (1991), 127-137.

[Dis] Diskant, V. I., Stability of the Solution of the Minkowski Equation (in Russian). Sibirsk. Mat. 14 (1973), 669-673, 696. English translation in Siberian Math. J. 14 (1973), 466-469. 
[Dur] R Durrett, Stochastic Calculus: A Practical Introduction Cambdidge university press, 2003.

[EK1] Eldan, R., Klartag, B., Approximately gaussian marginals and the hyperplane conjecture. Proc. of a workshop on "Concentration, Functional Inequalities and Isoperimetry", Contemporary Math., vol. 545, Amer. Math. Soc., (2011), 55-68.

[EK2] Eldan, R., Klartag, B., Dimensionality and the stability of the Brunn-Minkowski inequality. Annali SNS, 2011.

[F11] B. Fleury, Concentration in a thin euclidean shell for log-concave measures, J. Func. Anal. 259 (2010), 832841.

[Fl2] B. Fleury, Poincaré inequality in mean value for Gaussian polytopes, Probability theory and related fields, Volume 152, Numbers 1-2, 141-178.

[FMP1] Figalli, A., Maggi, F., Pratelli, A., A refined Brunn-Minkowski inequality for convex sets. Ann. Inst. H. Poincaré Anal. Non Linéaire, vol. 26, no. 6, (2009), 2511-2519.

[FMP2] Figalli, A., Maggi, F., Pratelli, A., A mass transportation approach to quantitative isoperimetric inequalities. Invent. Math., vol. 182, no. 1, (2010), 167- 211.

[Gu-M] O. Guedon, E. Milman, Interpolating thin-shell and sharp largedeviation estimates for isotropic log-concave measures, 2010

[Gr-M] M. Gromov and V. D. Milman. A topological application of the isoperimetric inequality. Amer. J. Math., 105(4):843854, 1983

[Groe] Groemer, H., On the Brunn-Minkowski theorem. Geom. Dedicata, vol. 27, no. 3, (1988), 357-371.

[Gros1] L. Gross Logarithmic Sobolev inequalities Amer. J. Math. 97 (1975), no. 4, 1061-1083

[Gros2] L. Gross, Logarithmic Sobolev inequalities and contractivity properties of semigroups, Dirichlet forms Varenna, 1992, 54-88, Lecture Notes in Math., 1563, Springer, Berlin, 1993.

[Leh] J. Lehec, Representation formula for the entropy and functional inequalities. arXiv: 1006.3028, 2010.

[KX] G. Kallianpur, J. Xiong, Stochastic Differential Equations in Infinite Dimensional Spaces Institute of mathematical statistics, lecture notes - monograph series. California, USA, 1995.

[K1] Klartag, B., A central limit theorem for convex sets. Invent. Math., 168, (2007), 91-131.

[K2] Klartag, B., Power-law estimates for the central limit theorem for convex sets. J. Funct. Anal., Vol. 245, (2007), 284-310.

[K3] Klartag, B., A Berry-Esseen type inequality for convex bodies with an unconditional basis. Probab. Theory Related Fields, vol. 145, no. 1-2, (2009), 1-33.

[K4] Klartag, B., Power-law estimates for the central limit theorem for convex sets. wherever, 2007. 
[KLS] R. Kannan, L. Lovász, and M. Simonovits. Isoperimetric problems for convex bodies and a localization lemma. Discrete Comput. Geom., 13(3-4):541559, 1995

[L] M. Ledoux, Spectral gap, logarithmic Sobolev constant, and geometric bounds. Surveys in differential geometry. Vol. IX, 219-240, Surv. Differ. Geom., IX, Int. Press, Somerville, MA, 2004.

[LV] L. Lovász and S. Vempala, The geometry of logconcave functions and sampling algorithms. Random Structures \& Algorithms, Vol. 30, no. 3, (2007), 307-358.

[Mil1] E. Milman, On the role of Convexity in Isoperimetry, SpectralGap and Concentration, Invent. Math. 177 (1), 1-43, 2009.

[Mil2] E. Milman, Isoperimetric Bounds on Convex Manifolds, Contemporary Math., proceedings of the Workshop on "Concentration,Functional Inequalities and Isoperimetry" in Florida, November 2009 .

[Ok] B. Oksendal Stochastic Differential Equations: An Introduction with Applications. Berlin: Springer. ISBN 3-540-04758-1, (2003).

[Oss] R. Osserman, Bonnesen-style isoperimetric inequalities. Amer. Math. Monthly, 86, no. 1, (1979), 1-29.

[Pis] G. Pisier, The volume of convex bodies and Banach space geometry. Cambridge Tracts in Mathematics, 94. Cambridge University Press, Cambridge, 1989.

[Seg] A. Segal, Remark on Stability of Brunn-Minkowski and Isoperimetric Inequalities for Convex Bodies. To appear in Gafa Seminar notes.

[Sud] V.N. Sudakov, Typical distributions of linear functionals in finitedimensional spaces of high dimension. (Russian) Dokl. Akad. Nauk SSSR 243 (1978), no. 6, 1402-1405.

[T] T. Tam, On Lei-Miranda-Thompson's result on singular values and diagonal elements. Linear Algebra and Its Applications, 272 (1998), 91-101.

[Vil] Villani, C., Topics in optimal transportation. Graduate Studies in Mathematics, 58. American Mathematical Society, Providence, RI, 2003.

e-mail address: roneneldan@gmail.com 